Youssef El-Khatib and Nicolas Privault (La Rochelle)

\title{
HEDGING IN COMPLETE MARKETS DRIVEN BY NORMAL MARTINGALES
}

Abstract. This paper aims at a unified treatment of hedging in market models driven by martingales with deterministic bracket $\langle M, M\rangle_{t}$, including Brownian motion and the Poisson process as particular cases. Replicating hedging strategies for European, Asian and Lookback options are explicitly computed using either the Clark-Ocone formula or an extension of the delta hedging method, depending on which is most appropriate.

1. Introduction. The Clark formula [Cla70] allows in principle the calculation of replicating hedging strategies in complete markets [KO91], but explicit computations are in general difficult to perform via this formula. For markets driven by Brownian motion a proof of the classical Black-Scholes formula via the Clark-Ocone formula can be found in [Øks96, Ch. 5, p. 13]. This method has recently been extended to markets driven by a Poisson process in [AOPU00]. Brownian motion and the compensated Poisson process share the important chaos representation property which is crucial for market completeness.

In this paper we consider a larger family $\left(M_{t}\right)_{t \in[0, T]}$ of martingales satisfying the following two conditions:

(a) the chaotic representation property (with respect to market completeness), i.e. every square-integrable functional, measurable with respect to the filtration generated by $\left(M_{t}\right)_{t \in[0, T]}$, can be expanded into a series of multiple stochastic integrals of deterministic functions with respect to $\left(M_{t}\right)_{t \in[0, T]}$,

(b) the condition $d\langle M, M\rangle_{t}=\alpha_{t}^{2} d t$, where $\left(\alpha_{t}\right)_{t \in[0, T]}$ is a square integrable deterministic function.

2000 Mathematics Subject Classification: 91B24, 91B26, 91B28, 60H05, $60 \mathrm{H} 07$.

Key words and phrases: normal martingales, chaos representation property, hedging strategies, exotic options. 
Hypothesis (b) implies that $\left([M, M]_{t}-\int_{0}^{t} \alpha_{s}^{2} d s\right)_{t \in[0, T]}$ is a martingale, hence from (a) there exists a process $\left(\phi_{t}\right)_{t \in[0, T]}$ such that $\left(M_{t}\right)_{t \in[0, T]}$ satisfies the structure equation

$$
d[M, M]_{t}=\alpha_{t}^{2} d t+\phi_{t} d M_{t}, \quad t \in[0, T]
$$

(cf. [É90]). This equation can be viewed as a decomposition of $d[M, M]_{t}$ by projection on $d t$ and $d M_{t}$, which yields a closed Itô type change of variable formula (see Prop. 2.3).

Brownian motion is obtained for $\alpha_{t}=1$ and $\phi_{t}=0$ for all $t \in[0, T]$, and the Poisson process corresponds to non-zero constant $\phi_{t}, t \in[0, T]$. The choice $\phi_{t}=\beta M_{t},-2 \leq \beta<0$, considered in [DP99], corresponds to the Azéma martingale and yields another complete market model with jumps. Choosing $\left(\phi_{t}\right)_{t \in[0, T]}$ to be a deterministic function allows the driving process to be alternatively Brownian or Poisson, depending on the vanishing of $\phi_{t}$ (see [JP02] for the corresponding market model).

The Clark-Ocone formula states the predictable representation of a random variable $F$ as

$$
F=E[F]+\int_{0}^{T} E\left[D_{t} F \mid \mathcal{F}_{t}\right] d M_{t},
$$

where $\left(\mathcal{F}_{t}\right)_{t \in[0, T]}$ is the filtration generated by $\left(M_{t}\right)_{t \in[0, T]}$ and $D_{t}$ is the gradient operator that lowers the degree of multiple stochastic integrals with respect to $\left(M_{t}\right)_{t \in[0, T]}$. One of the goals of this paper is to compute the process $t \mapsto E\left[D_{t} F \mid \mathcal{F}_{t}\right]$ in several situations. We obtain explicit hedging formulas for European calls in the mixed Brownian-Poisson model of [JP02] and in the Azéma martingale model of [DP99] and for Asian and Lookback options.

More precisely, let $\left(S_{t, T}^{x}\right)_{t \in[0, T]}$ denote the stock price process driven by $\left(M_{t}\right)_{t \in[0, T]}$, starting from $x$ at time $t$, with volatility $\left(\sigma_{t}\right)_{t \in[0, T]}$, and let $i_{t}=1_{\left\{\phi_{t}=0\right\}}, j_{t}=1-i_{t}, S_{t}=S_{0, t}^{1}$ for $t \in[0, T]$. In a model with deterministic structure equation, i.e. $d[M, M]_{t}=\alpha_{t}^{2} d t+\phi_{t} d M_{t}$ with deterministic $\left(\phi_{t}\right)_{t \in[0, T]}$, the replicating hedging strategy of a European call with payoff $\left(S_{T}-K\right)^{+}$is given by

$$
\begin{aligned}
t \mapsto \frac{e^{-\int_{t}^{T} r_{s} d s}}{\sigma_{t} S_{t}} E[ & i_{t} \sigma_{t} S_{t, T}^{x} 1_{\left\{S_{t, T}^{x} \geq K\right\}} \\
& \left.\quad+\frac{j_{t}}{\phi_{t}}\left(\sigma_{t} \phi_{t} S_{t, T}^{x}-\left(K-S_{t, T}^{x}\right)^{+}\right) 1_{\left\{S_{t, T}^{x} \geq K /\left(1+\sigma_{t}\right)\right\}}\right]_{x=S_{t}}
\end{aligned}
$$

(cf. (4.1.6) and Prop. 4.1). This formula extends both the classical BlackScholes hedging formula in the Brownian case $\left(\phi_{t}=0\right.$ for all $\left.t \in[0, T]\right)$, and the hedging formula of [AOPU00, Th. 6.1] in the Poissonian jump case 
( $\phi_{t}=1$ for all $t \in[0, T]$ ). It can be obtained both from the Clark formula and from martingale methods. The above conditional expectation can also be explicitly computed (see Prop. 4.2). The case of Asian options is treated in Proposition 4.3 in the deterministic structure equation model, and Lookback options are considered in Proposition 4.5 in a market driven by Brownian motion. In the Azéma martingale model of [DP99], i.e. $d[M, M]_{t}=d t+$ $\beta M_{t^{-}} d M_{t},-2 \leq \beta<0$, we obtain

$$
\begin{aligned}
t \mapsto \frac{e^{-\int_{t}^{T} r_{s} d s}}{\beta M_{t} \sigma_{t} S_{t}} E\left[\sigma_{t} \beta(y+\right. & \left.M_{T}-M_{t}\right) S_{t, T}^{x} 1_{\left[\frac{K}{1+\sigma_{t} \beta\left(y+M_{T}-M_{t}\right)}, \infty[\right.}\left(S_{t, T}^{x}\right) \\
& \left.+\left(S_{t, T}^{x}-K\right) 1_{\left[\frac{K}{1+\sigma_{t} \beta\left(y+M_{T}-M_{t}\right)}, K\right]}\left(S_{t, T}^{x}\right)\right]_{x=S_{t}}^{y=M_{t}}
\end{aligned}
$$

(see Prop. 4.4).

We proceed as follows. In Section 2 we introduce the notation of chaotic calculus, the solutions of structure equations and the Clark-Ocone formula which gives the predictable representation of the random variable $F$. We also state the change of variable formula and Girsanov theorem, which hold in a particular form for solutions of structure equations. In Section 3 we describe different methods for the computation of predictable representations for the general class of normal martingales having the chaos representation property. The intrinsic expression of the gradient $D$ is completely known if $\left(\phi_{t}\right)_{t \in[0, T]}$ is deterministic, i.e. for the Brownian, Poisson and deterministic structure equation models (Section 3.2). In the Markovian case (Section 3.3) it is possible to combine the Clark-Ocone and Itô formulas to obtain the explicit predictable representation of $F$. Section 4 is devoted to the computation of replicating portfolios. In Section 4.2 we hedge European calls using the Clark formula, extending the method applied in the Brownian case in [Øks96, Ch. 5, pp. 13-15]. In Sections 4.3-4.5 we deal with Asian, European and Lookback options, in particular we use the delta hedging approach to recover some results obtained in [Ber98] from the Clark formula.

\section{Notation and preliminaries}

2.1. Chaotic calculus. Let $\left(M_{t}\right)_{t \in[0, T]}$ be a martingale on the space $\Omega$ of càdlàg functions from $[0, T]$ to $\mathbb{R}$, having the chaos representation property. Let $\left(\alpha_{t}\right)_{t \in[0, T]} \in L^{2}([0, T])$ be a positive deterministic non-vanishing square-integrable function, and assume that $\left(M_{t}\right)_{t \in[0, T]}$ has deterministic angle bracket $d\langle M, M\rangle_{t}=\alpha_{t}^{2} d t$. We denote by $\left(\mathcal{F}_{t}\right)_{t \in[0, T]}$ the filtration generated by $\left(M_{t}\right)_{t \in[0, T]}$, and by $L_{\alpha}^{2}([0, T])^{\circ n}$ the space $L^{2}\left([0, T], \alpha_{t}^{2} d t\right)^{\circ n}$ of $\alpha_{t_{1}}^{2} \ldots \alpha_{t_{n}}^{2} d t_{1} \ldots d t_{n}$ square-integrable symmetric functions. The multiple stochastic integral $I_{n}\left(f_{n}\right)$ is defined as 


$$
I_{n}\left(f_{n}\right)=n ! \int_{0}^{T} \int_{0}^{t_{n}} \ldots \int_{0}^{t_{2}} f_{n}\left(t_{1}, \ldots, t_{n}\right) d M_{t_{1}} \ldots d M_{t_{n}}, \quad n \geq 1
$$

for $f_{n} \in L_{\alpha}^{2}([0, T])^{\circ n}$, with

$$
E\left[I_{n}\left(f_{n}\right) I_{m}\left(g_{m}\right)\right]=n ! 1_{\{n=m\}}\left\langle f_{n}, g_{m}\right\rangle_{L_{\alpha}^{2}([0, T])^{\circ n}} .
$$

The chaos representation property for $\left(M_{t}\right)_{t \in[0, T]}$ states that every $F \in$ $L^{2}(\Omega)$ has a decomposition as $F=\sum_{n=0}^{\infty} I_{n}\left(f_{n}\right)$. Let $D: \operatorname{Dom}(D) \rightarrow$ $L^{2}\left(\Omega \times[0, T], d P \times \alpha_{t}^{2} d t\right)$ denote the closable, unbounded gradient operator defined as

$$
D_{t} F=\sum_{n=1}^{\infty} n I_{n-1}\left(f_{n}(*, t)\right) \quad d P \times d t \text {-a.e. }
$$

with $F=\sum_{n=0}^{\infty} I_{n}\left(f_{n}\right)$ in $\operatorname{Dom}(D)$, i.e.

$$
\sum_{n=1}^{\infty} n n !\left\|f_{n}\right\|_{L^{2}\left(\mathbb{R}_{+}^{n}\right)}^{2}<\infty .
$$

2.2. Structure equations. Let $L_{\text {ad }}^{\infty}(\Omega \times[0, T])$ be the space of bounded, $\left(\mathcal{F}_{t}\right)_{t \in \mathbb{R}_{+}}$-adapted stochastic processes. We assume that $\left(M_{t}\right)_{t \in[0, T]}$ is a solution of the structure equation

$$
d[M, M]_{t}=\alpha_{t}^{2} d t+\phi_{t} d M_{t}, \quad t \in[0, T],
$$

where $\phi_{t}=\varphi\left(t, M_{t^{-}}\right)$is a deterministic function of $t$ and $M_{t}$. Existence and uniqueness of solutions are guaranteed when $\phi_{t}$ is a deterministic function [É90]. Existence is proved when $\phi_{t}=\varphi\left(M_{t^{-}}\right)$and $\varphi$ is a continuous function [Mey89], and the solution is unique when $\varphi(x)=\beta x$ with $\beta \in[-2,0)$ (cf. [É90]). See also [Pha00], [Tav99] for recent results on structure equations. Let $i_{t}=1_{\left\{\phi_{t}=0\right\}}$ and $j_{t}=1_{\left\{\phi_{t} \neq 0\right\}}=1-i_{t}$ for $t \in[0, T]$. The continuous part of $\left(M_{t}\right)_{t \in[0, T]}$ is given by $d M_{t}^{c}=i_{t} d M_{t}$ and the possible jump of $\left(M_{t}\right)_{t \in[0, T]}$ at time $t \in[0, T]$ is $\Delta M_{t}=\phi_{t}($ see $[$ É90, p. 77]).

(a) If $\left(\phi_{t}\right)_{t \in[0, T]}$ is deterministic, let $\lambda_{t}=j_{t} \alpha_{t}^{2} / \phi_{t}^{2}$ for $t \in[0, T]$. Then $\left(M_{t}\right)_{t \in[0, T]}$ can be represented as

$$
d M_{t}=i_{t} \alpha_{t} d B_{t}+\phi_{t}\left(d N_{t}-\lambda_{t} d t\right), \quad t \in[0, T], \quad M_{0}=0,
$$

where $\left(B_{t}\right)_{t \in[0, T]}$ is a standard Brownian motion, and $\left(N_{t}\right)_{t \in[0, T]}$ a Poisson process independent of $\left(B_{t}\right)_{t \in[0, T]}$, with intensity $\nu_{t}=\int_{0}^{t} \lambda_{s} d s$ for $t \in[0, T]$ (cf. [É90, Prop. 4] and [JP02]).

(b) If $\phi_{t}=\beta M_{t}, \beta \in \mathbb{R}$, then (2.2.1) has a unique solution called the Azéma martingale (cf. [É90]). If $-2 \leq \beta<0$, this solution has the chaos representation property and it has been used to model a complete market with jumps in [DP99]. 
Figure 1 shows a sample path of $\left(S_{t}\right)_{t \in[0, T]}$ and the corresponding function $\left(i_{t}\right)_{t \in[0, T]}$ chosen to be a simple indicator function, with $S_{0}=4, \sigma_{t}=1$, $\alpha_{t}^{2}=50, \phi_{t}=1.6 i_{t}$, for $t \in[0, T]$.

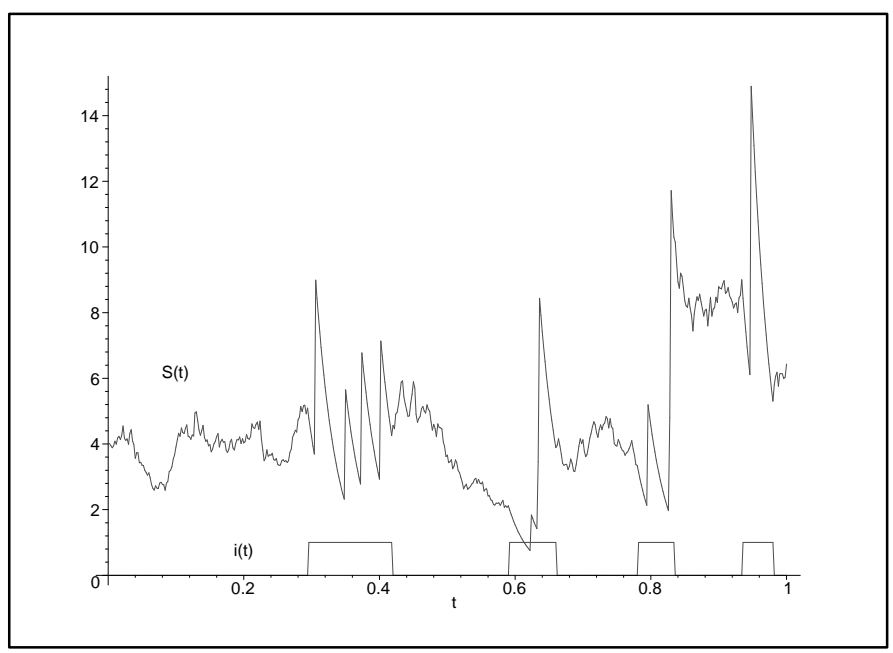

Fig. 1. Sample trajectory of $\left(S_{t}\right)_{t \in[0, T]}$ (vertical lines represent jumps)

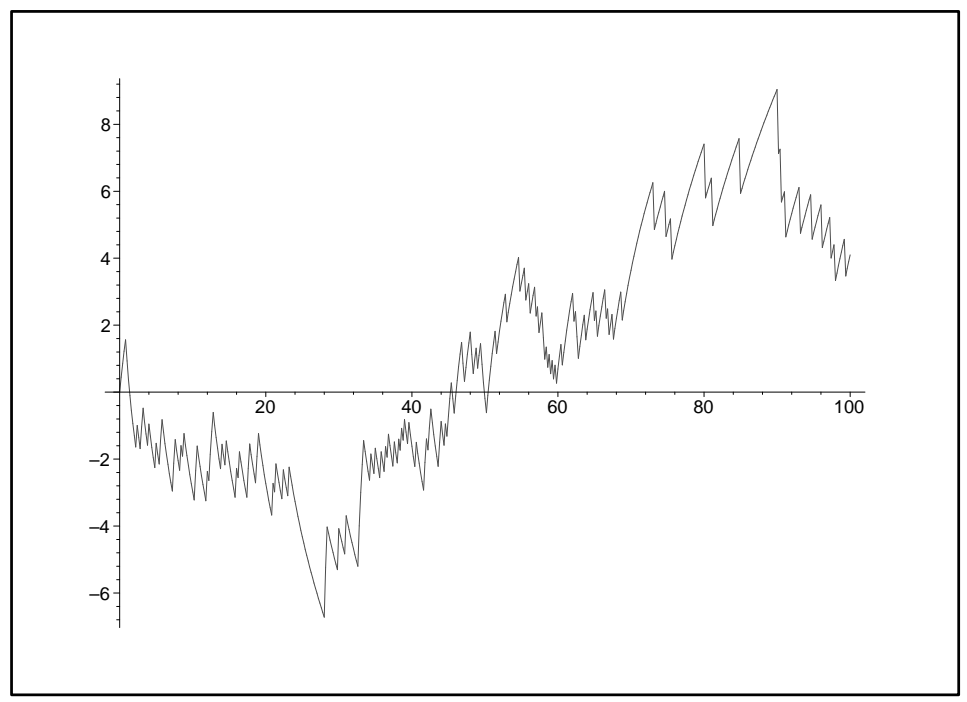

Fig. 2. Sample path of an Azéma martingale

Figure 2 is a simulation of an Azéma martingale with $\beta \neq-1$, from a discretization of the structure equation (2.2.1): 
with probabilities

$$
\Delta X_{t}=\frac{\beta X_{t^{-}}}{2} \pm \sqrt{\left(\beta X_{t^{-}} / 2\right)^{2}+\Delta t},
$$

$$
p=\frac{1}{2} \mp \frac{\beta X_{t^{-}}}{\sqrt{\left(\beta X_{t^{-}} / 2\right)^{2}+\Delta t}} .
$$

In all cases of interest in this paper we have $\left(\phi_{t}\right)_{t \in[0, T]} \in L_{\mathrm{ad}}^{\infty}(\Omega \times[0, T])$, since if $\left(\phi_{t}\right)_{t \in[0, T]}=\left(\beta M_{t}\right)_{t \in[0, T]}$ then $\sup _{t \in[0, T]}\left|M_{t}\right| \leq(-2 / \beta)^{1 / 2}$ (see [É90, p. 83]). Given $\left(u_{t}\right)_{t \in[0, T]} \in L_{\mathrm{ad}}^{\infty}(\Omega \times[0, T])$, we denote by $\left(\xi_{t}(u)\right)_{t \in[0, T]}$ the solution of the equation

$$
Z_{t}=1+\int_{0}^{t} Z_{s^{-}} u_{s} d M_{s}, \quad t \in[0, T]
$$

which can be written as ([Pro90, Th. 36, p. 77])

$$
\xi_{t}(u)=\exp \left(\int_{0}^{t} u_{s} d M_{s}-\frac{1}{2} \int_{0}^{t} u_{s}^{2} \alpha_{s}^{2} i_{s} d s\right) \prod_{s \in J_{M}^{t}}\left(1+u_{s} \phi_{s}\right) e^{-u_{s} \phi_{s}},
$$

where $J_{M}^{t}$ denotes the set of jump times of $\left(M_{s}\right)_{s \in[0, t]}$ for $t \in[0, T]$, and let $\xi(u)=\xi_{T}(u)$. If $u \in L^{\infty}([0, T])$ then $\xi_{t}(u)$ can be represented as

$$
\xi_{t}(u)=\sum_{n=0}^{\infty} \frac{1}{n !} I_{n}\left(\left(u 1_{[0, t]}\right)^{\otimes n}\right),
$$

and we have $D_{s} \xi_{t}(u)=1_{[0, t]}(s) u_{s} \xi_{t}(u)$ for $s, t \in[0, T]$.

Definition 2.1. Let $\mathcal{S}$ denote the linear space generated by exponential vectors of the form $\xi(u)$, where $u \in L^{\infty}([0, T])$.

The space $\mathcal{S}$ is dense in $L^{2}(\Omega)$, and by the lemma below, $\mathcal{S}$ is an algebra for the pointwise multiplication of random variables if $\left(\phi_{t}\right)_{t \in[0, T]}$ is deterministic. The following is a version of Yor's formula [Yor76] or Theorem 37 of [Pro90, p. 79], for martingales with deterministic bracket $\langle M, M\rangle_{t}$.

Lemma 2.2. For any $u, v \in L^{\infty}([0, T])$,

$$
\xi(u) \xi(v)=\exp \left(\langle u, v\rangle_{L_{\alpha}^{2}([0, T])}\right) \xi(u+v+\phi u v) .
$$

Proof. For $u, v \in L^{\infty}([0, T])$ we have

$$
\begin{aligned}
d\left(\xi_{t}(u) \xi_{t}(v)\right)= & u_{t} \xi_{t^{-}}(u) \xi_{t^{-}}(v) d M_{t}+v_{t} \xi_{t^{-}}(v) \xi_{t^{-}}(u) d M_{t} \\
& +v_{t} u_{t} \xi_{t^{-}}(v) \xi_{t^{-}}(u) d[M, M]_{t} \\
= & u_{t} \xi_{t^{-}}(u) \xi_{t^{-}}(v) d M_{t}+v_{t} \xi_{t^{-}}(v) \xi_{t^{-}}(u) d M_{t}+v_{t} u_{t} \xi_{t}(v) \xi_{t}(u) \alpha_{t}^{2} d t \\
& +\phi_{t} u_{t} v_{t} \xi_{t^{-}}(v) \xi_{t^{-}}(u) d M_{t} \\
= & v_{t} u_{t} \xi_{t}(v) \xi_{t}(u) \alpha_{t}^{2} d t+\xi_{t^{-}}(v) \xi_{t^{-}}(u)\left(u_{t}+v_{t}+\phi_{t} u_{t} v_{t}\right) d M_{t} .
\end{aligned}
$$


Hence

$d\left(e^{-\int_{0}^{t} u_{s} v_{s} \alpha_{s}^{2} d s} \xi_{t}(u) \xi_{t}(v)\right)=e^{-\int_{0}^{t} u_{s} v_{s} \alpha_{s}^{2} d s} \xi_{t^{-}}(v) \xi_{t^{-}}(u)\left(u_{t}+v_{t}+\phi_{t} u_{t} v_{t}\right) d M_{t}$, which shows that $\exp \left(-\langle u, v\rangle_{L_{\alpha}^{2}([0, T])}\right) \xi(u) \xi(v)=\xi(u+v+\phi u v)$. Relation (2.2.5) follows then by comparison with $(2.2 .3)$.

2.3. Change of variable formula. We recall the following change of variable formula, which follows from Proposition 2 of [É90] after addition of an absolutely continuous drift term.

Proposition 2.3. Let $X=\left(X_{t}\right)_{t \in[0, T]}$ be an $\mathbb{R}^{n}$-valued process satisfying

$$
d X_{t}=R_{t} d t+K_{t} d M_{t}, \quad X_{0}>0
$$

where $R=\left(R_{t}\right)_{t \in[0, T]}$ and $K=\left(K_{t}\right)_{t \in[0, T]}$ are two predictable squareintegrable $\mathbb{R}^{n}$-valued processes. For any function $\mathbb{R}_{+} \times \mathbb{R}^{n} \ni(t, x) \mapsto f_{t}(x)$ in $\mathcal{C}_{\mathrm{b}}^{2}\left(\mathbb{R}_{+} \times \mathbb{R}^{n} ; \mathbb{R}\right)$ we have

$$
f_{t}\left(X_{t}\right)=f_{0}\left(X_{0}\right)+\int_{0}^{t} L_{s} f_{s}\left(X_{s}\right) d M_{s}+\int_{0}^{t} U_{s} f_{s}\left(X_{s}\right) d s+\int_{0}^{t} \frac{\partial f_{s}}{\partial s}\left(X_{s}\right) d s
$$

where

$$
L_{s} f_{s}\left(X_{s}\right)=i_{s}\left\langle K_{s}, \nabla f_{s}\left(X_{s}\right)\right\rangle+\frac{j_{s}}{\phi_{s}}\left(f_{s}\left(X_{s^{-}}+\phi_{s} K_{s^{-}}\right)-f_{s}\left(X_{s^{-}}\right)\right)
$$

and

$$
\begin{aligned}
U_{s} f_{s}\left(X_{s}\right)= & R_{s} \nabla f_{s}\left(X_{s}\right)+\alpha_{s}^{2}\left(\frac{1}{2} i_{s}\left\langle\operatorname{Hess} f_{s}\left(X_{s}\right), K_{s} \otimes K_{s}\right\rangle\right. \\
& \left.+\frac{j_{s}}{\phi_{s}^{2}}\left(f_{s}\left(X_{s^{-}}+\phi_{s} K_{s^{-}}\right)-f_{s}\left(X_{s^{-}}\right)-\phi_{s}\left\langle K_{s}, \nabla f_{s}\left(X_{s}\right)\right\rangle\right)\right)
\end{aligned}
$$

with the convention $0 / 0=0$.

2.4. Girsanov theorem. The Girsanov theorem holds in a particular form when $\left(M_{t}\right)_{t \in[0, T]}$ is the solution of a structure equation (1.0.1). Let $\left(\psi_{t}\right)_{t \in[0, T]}$ be a bounded predictable process such that $1+\phi_{t} \psi_{t}>0$ for all $t \in[0, T]$, let $\left(l_{t}\right)_{t \in[0, T]}$ denote the solution of the equation

$$
d l_{t}=l_{t^{-}} \psi_{t} d M_{t}, \quad t \in[0, T], \quad l_{0}=1,
$$

and let $Q$ be the probability defined by

$$
l_{t}=E\left[\frac{d Q}{d P} \mid \mathcal{F}_{t}\right], \quad t \in[0, T]
$$

Proposition 2.4. Under the probability $Q$, the process

$$
Z_{t}=M_{t}-\int_{0}^{t} \alpha_{s}^{2} \psi_{s} d s, \quad t \in[0, T]
$$


is a local martingale which satisfies the structure equation

$$
d[Z, Z]_{t}=\alpha_{t}^{2}\left(1+\phi_{t} \psi_{t}\right) d t+\phi_{t} d Z_{t}, \quad t \in[0, T] .
$$

Proof. From the Girsanov theorem,

$$
d Z_{t}=d M_{t}-\frac{1}{l_{t^{-}}} d\langle L, M\rangle_{t}=d M_{t}-\alpha_{t}^{2} \psi_{t} d t
$$

is a local martingale under $Q$, with $d\langle L, M\rangle_{t}=l_{t^{-}} \alpha_{t}^{2} \psi_{t} d t$, and

$$
d[Z, Z]_{t}=d[M, M]_{t}=\alpha_{t}^{2} d t+\phi_{t} d M_{t}=\alpha_{t}^{2}\left(1+\phi_{t} \psi_{t}\right) d t+\phi_{t} d Z_{t}
$$

If $\left(\psi_{t}\right)_{t \in[0, T]}$ and $\left(\phi_{t}\right)_{t \in[0, T]}$ are deterministic, then $\left(Z_{t}\right)_{t \in[0, T]}$ has the chaos representation property under $Q$, since (2.4.3) is a deterministic structure equation.

\section{Computations of predictable representations}

3.1. Clark formula. The Clark-Ocone formula (cf. [Cla70], [KO91]) is a consequence of the chaos representation property for $\left(M_{t}\right)_{t \in[0, T]}$, and states that any $F \in \operatorname{Dom}(D) \subset L^{2}\left(\Omega, \mathcal{F}_{T}, P\right)$ has a representation

$$
F=E[F]+\int_{0}^{T} E\left[D_{t} F \mid \mathcal{F}_{t}\right] d M_{t} .
$$

It can be proved as follows:

$$
\begin{aligned}
F & =E[F]+\sum_{n=1}^{\infty} n ! \int_{0}^{T} \int_{0}^{t_{n}} \ldots \int_{0}^{t_{2}} f_{n}\left(t_{1}, \ldots, t_{n}\right) d M_{t_{1}} \ldots d M_{t_{n}} \\
& =E[F]+\sum_{n=1}^{\infty} n \int_{0}^{T} I_{n-1}\left(f_{n}(*, t) 1_{\{* \leq t\}}\right) d M_{t} \\
& =E[F]+\int_{0}^{T} E\left[D_{t} F \mid \mathcal{F}_{t}\right] d M_{t} .
\end{aligned}
$$

Although $D: L^{2}\left(\Omega, \mathcal{F}_{T}, P\right) \rightarrow L^{2}\left(\Omega \times[0, T], d P \times \alpha_{t}^{2} d t\right)$ is unbounded, the representation formula (3.1.1) can be extended to $F \in L^{2}\left(\Omega, \mathcal{F}_{T}, P\right)$.

Proposition 3.1. The operator $F \mapsto E[D . F \mid \mathcal{F}$. $]$ taking its values in the space of square-integrable adapted processes has a continuous extension from $\operatorname{Dom}(D)$ to $L^{2}\left(\Omega, \mathcal{F}_{T}, P\right)$.

Proof. We use the bound

$$
\begin{aligned}
\|E[D . F \mid \mathcal{F} .]\|_{L^{2}(\Omega \times[0, T])}^{2} & =\|E[F]-F\|_{L^{2}(\Omega)}^{2}=\operatorname{var}(F) \\
& \leq\|F\|_{L^{2}(\Omega)}^{2}, \quad F \in \operatorname{Dom}(D) .
\end{aligned}
$$


Instead of the adapted projection $\left(E\left[D_{t} F \mid \mathcal{F}_{t}\right]\right)_{t \in[0, T]}$ one may also use the predictable projection $\left(E\left[D_{t} F \mid \mathcal{F}_{t^{-}}\right]\right)_{t \in[0, T]}$. Here this leads to the same representation since both processes coincide in $L^{2}\left(\Omega \times[0, T], d P \times \alpha_{t}^{2} d t\right)$.

3.2. Deterministic structure equation. In this subsection we consider the case where $\left(\phi_{t}\right)_{t \in[0, T]} \in L^{\infty}([0, T])$ is a deterministic function. In this case, the probabilistic interpretation of $D_{t}$ is known and $D_{t} F$ is explicitly computable. We define the operator $D^{B}: \mathcal{S} \rightarrow L^{2}\left(\Omega \times \mathbb{R}_{+}, d P \times \alpha_{t}^{2} d t\right)$ on $\mathcal{S}$ as

$$
\left\langle D^{B} F, u\right\rangle_{L_{\alpha}^{2}([0, T])}=\left.\frac{d}{d \varepsilon} F\left(\omega(\cdot)+\varepsilon \int_{0} i_{s} u_{s} d s\right)\right|_{\varepsilon=0}, \quad F \in \mathcal{S} .
$$

For $F=\xi(u)$ and $g \in L_{\alpha}^{2}([0, T])$ we have

$$
\begin{aligned}
\left\langle D^{B} F, g\right\rangle_{L_{\alpha}^{2}([0, T])} & =\left.\frac{d}{d \varepsilon} \exp \left(\varepsilon \int_{0}^{T} g_{s} u_{s} \alpha_{s} i_{s} d s\right) \xi(u)\right|_{\varepsilon=0} \\
& =\int_{0}^{T} g_{s} u_{s} \alpha_{s} i_{s} d s \xi(u),
\end{aligned}
$$

hence $D_{t}^{B} \xi(u)=i_{t} u_{t} \xi(u)$ for $t \in[0, T]$, where

$$
\xi(u)=\exp \left(\int_{0}^{T} u_{s} d M_{s}-\frac{1}{2} \int_{0}^{T} u_{s}^{2} \alpha_{s}^{2} i_{s} d s\right) \prod_{s \in J_{M}^{T}}\left(1+u_{s} \phi_{s}\right) e^{-u_{s} \phi_{s}} .
$$

Note that the definition of $D^{B} F$ by duality in $L_{\alpha}^{2}([0, T])$ implies

$$
D_{t}^{B} \int_{0}^{T} u_{s} \alpha_{s} d B_{s}=u_{t}, \quad t \in[0, T]
$$

We define a linear transformation $T_{t}^{\phi}$ of exponential vectors, and more generally of elements of $\mathcal{S}$, as

$$
T_{t}^{\phi} \xi(u)=\left(1+u_{t} \phi_{t}\right) \xi(u), \quad u \in L^{\infty}([0, T]) .
$$

The transformation $T_{t}^{\phi}$ is well defined on $\mathcal{S}$ because $\xi\left(u_{1}\right), \ldots, \xi\left(u_{n}\right)$ are linearly independent if $u_{1}, \ldots, u_{n}$ are distinct elements of $L^{2}\left(\mathbb{R}_{+}\right)$. Since $\Delta M_{t}=0 d t \times d P$-a.e., $T_{t}^{\phi} \xi(u)$ coincides $d t \times d P$-a.e. with the value at time $T$ of the solution of the equation

$$
Z_{s}^{t}=1+\int_{0}^{s} Z_{\tau^{-}}^{t} u_{\tau} d M_{\tau}^{t}, \quad s \in[0, T]
$$

where $\left(M_{s}^{t}\right)_{s \in[0, T]}$ is defined as

$$
M_{s}^{t}=M_{s}+\phi_{t} 1_{[t, T]}(s), \quad s \in[0, T] .
$$


In order to see this we check that $Z_{s}^{t}=\xi_{s}(u)$ for $s<t$,

$$
Z_{t}^{t}=\left(1+\phi_{t} u_{t}\right) Z_{t^{-}}^{t}=\left(1+\phi_{t} u_{t}\right) \xi_{t^{-}}(u)=\left(1+\phi_{t} u_{t}\right) \xi_{t}(u)
$$

(since $\xi_{t^{-}}(u)=\xi_{t}(u)$ a.s. for fixed $t$ ), and for $s>t$,

$$
Z_{s}^{t}=Z_{t}^{t}+\int_{t}^{s} Z_{\tau^{-}}^{t} u_{\tau} d M_{\tau}=\left(1+\phi_{t} u_{t}\right) \xi_{t}(u)+\int_{t}^{s} Z_{\tau^{-}}^{t} u_{\tau} d M_{\tau}
$$

hence

$$
\frac{Z_{s}^{t}}{1+\phi_{t} u_{t}}=\xi_{t}(u)+\int_{t}^{s} \frac{Z_{\tau^{-}}^{t}}{1+\phi_{t} u_{t}} u_{\tau} d M_{\tau}, \quad s>t,
$$

which implies, from (2.2.3),

$$
\frac{Z_{s}^{t}}{1+\phi_{t} u_{t}}=\xi_{s}(u), \quad s>t,
$$

and $Z_{T}^{t}=\left(1+\phi_{t} u_{t}\right) \xi(u)=T_{t}^{\phi} \xi(u) P$-a.s. for $t \in[0, T]$.

In other terms, $T_{t}^{\phi} F, F \in \mathcal{S}$, can be interpreted as the evaluation of $F$ on the trajectories of $\left(M_{s}\right)_{s \in[0, T]}$ perturbed by addition of a jump of height $\phi_{t}$ at time $t$.

Proposition 3.2. The transformation $T_{t}^{\phi}$ is multiplicative, i.e.

$$
T_{t}^{\phi}(F G)=\left(T_{t}^{\phi} F\right)\left(T_{t}^{\phi} G\right), \quad F, G \in \mathcal{S} .
$$

Moreover,

$$
D_{t} F=D_{t}^{B} F+\frac{j_{t}}{\phi_{t}}\left(T_{t}^{\phi} F-F\right), \quad t \in[0, T], F \in \mathcal{S},
$$

and

$$
D_{t}(F G)=F D_{t} G+G D_{t} F+\phi_{t} D_{t} F D_{t} G, \quad t \in[0, T], F, G \in \mathcal{S} .
$$

Proof. For the multiplicativity we note that

$$
\begin{aligned}
T_{t}^{\phi}(\xi(u) \xi(v)) & =\exp \left(\langle u, v\rangle_{L_{\alpha}^{2}([0, T])}\right) T_{t}^{\phi} \xi(u+v+\phi u v) \\
& =\exp \left(\langle u, v\rangle_{L_{\alpha}^{2}([0, T])}\right)\left(1+\phi_{t}\left(u_{t}+v_{t}+\phi_{t} u_{t} v_{t}\right)\right) \xi(u+v+\phi u v) \\
& =\left(1+\phi_{t} u_{t}\right)\left(1+\phi_{t} v_{t}\right) \xi(u) \xi(v) \\
& =T_{t}^{\phi} \xi(u) T_{t}^{\phi} \xi(v) .
\end{aligned}
$$

When $\phi_{t}=0$ we have $D_{t}^{B} F=i_{t} u_{t} \xi(u)=i_{t} D_{t} F$, hence

$$
\begin{aligned}
D_{t} \xi(u) & =i_{t} D_{t} \xi(u)+j_{t} D_{t} \xi(u)=i_{t} u_{t} \xi(u)+j_{t} u_{t} \xi(u) \\
& =D_{t}^{B} \xi(u)+\frac{j_{t}}{\phi_{t}}\left(T_{t}^{\phi} \xi(u)-\xi(u)\right) \quad t \in[0, T] .
\end{aligned}
$$


Concerning the product rule we have, from Lemma 2.2,

$$
\begin{aligned}
D_{t}(\xi(u) \xi(v)) & =\exp \left(\int_{0}^{T} u_{s} v_{s} \alpha_{s}^{2} d s\right) D_{t} \xi(u+v+\phi u v) \\
& =\exp \left(\int_{0}^{T} u_{s} v_{s} \alpha_{s}^{2} d s\right)\left(u_{t}+v_{t}+\phi_{t} u_{t} v_{t}\right) \xi(u+v+\phi u v) \\
& =\left(u_{t}+v_{t}+\phi_{t} u_{t} v_{t}\right) \xi(u) \xi(v) \\
& =\xi(u) D_{t} \xi(v)+\xi(v) D_{t} \xi(u)+\phi_{t} D_{t} \xi(u) D_{t} \xi(v)
\end{aligned}
$$

for $u, v \in L^{\infty}([0, T])$ (see also [Pri96, (6)].

If $\left(\phi_{t}\right)_{t \in[0, T]}$ is random the probabilistic interpretation of $D$ is unknown, but we have the product rule

$$
\begin{aligned}
E\left[D_{t}(F G) \mid \mathcal{F}_{t}\right]= & E\left[F D_{t} G \mid \mathcal{F}_{t}\right]+E\left[G D_{t} F \mid \mathcal{F}_{t}\right] \\
& +\phi_{t} E\left[D_{t} F D_{t} G \mid \mathcal{F}_{t}\right]
\end{aligned}
$$

for $F, G \in \mathcal{S}$ and $t \in[0, T]$ (cf. [PSV00, Prop. 5]).

3.3. Markovian case. This section presents a representation method which is based on the Itô formula and the Markov property (see also [Pro01] in the continuous case). Let $\left(X_{t}\right)_{t \in[0, T]}$ be an $\mathbb{R}^{n}$-valued Markov (not necessarily time homogeneous) process defined on $\Omega$, satisfying a change of variable formula of the form

$$
f\left(X_{t}\right)=f\left(X_{0}\right)+\int_{0}^{t} L_{s} f\left(X_{s}\right) d M_{s}+\int_{0}^{t} U_{s} f\left(X_{s}\right) d s, \quad t \in[0, T],
$$

where $L_{s}, U_{s}$ are operators defined on $\mathcal{C}^{2}$ functions. We assume that the semigroup $\left(P_{s, t}\right)_{0 \leq s \leq t \leq T}$ associated to $\left(X_{t}\right)_{t \in[0, T]}$, i.e.

$$
P_{s, t} f\left(X_{s}\right)=E\left[f\left(X_{t}\right) \mid \mathcal{F}_{s}\right]=E\left[f\left(X_{t}\right) \mid X_{s}\right], \quad 0 \leq s \leq t \leq T,
$$

acts on $\mathcal{C}_{\mathrm{b}}^{2}\left(\mathbb{R}^{n}\right)$ functions, with $P_{s, t} \circ P_{t, u}=P_{s, u}$ for $0 \leq s \leq t \leq u \leq T$. Although the probabilistic interpretation of $D$ is not known when $\left(\phi_{t}\right)_{t \in[0, T]}$ is random, it is still possible to compute the explicit predictable representation of $f\left(X_{T}\right)$ using the Itô formula and the Markov property.

Lemma 3.3. Let $f \in \mathcal{C}_{\mathrm{b}}^{2}\left(\mathbb{R}^{n}\right)$. Then

$$
E\left[D_{t} f\left(X_{T}\right) \mid \mathcal{F}_{t}\right]=\left(L_{t}\left(P_{t, T} f\right)\right)\left(X_{t}\right), \quad t \in[0, T] .
$$

Proof. We apply the change of variable formula (3.3.1) to $t \mapsto P_{t, T} f\left(X_{t}\right)$ $=E\left[f\left(X_{T}\right) \mid \mathcal{F}_{t}\right]$, since $P_{t, T} f$ is $\mathcal{C}^{2}$. Using the fact that the finite variation term vanishes since $t \mapsto P_{t, T} f\left(X_{t}\right)$ is a martingale (see e.g. [Pro90, Cor. 1, 
p. 64]), we obtain

$$
P_{t, T} f\left(X_{t}\right)=P_{0, T} f\left(X_{0}\right)+\int_{0}^{t}\left(L_{s}\left(P_{s, T} f\right)\right)\left(X_{s}\right) d M_{s}, \quad t \in[0, T],
$$

with $P_{0, T} f\left(X_{0}\right)=E\left[f\left(X_{T}\right)\right]$. Letting $t=T$, we obtain (3.3.2) by uniqueness of the representation (3.1.1) applied to $F=f\left(X_{T}\right)$.

In practice, we will use Proposition 3.1 to extend $\left(E\left[D_{t} f\left(X_{T}\right) \mid \mathcal{F}_{t}\right]\right)_{t \in[0, T]}$ to a less regular function $f: \mathbb{R}^{n} \rightarrow \mathbb{R}$. As an example, if $\phi_{t}$ is written as $\phi_{t}=$ $\varphi\left(t, M_{t}\right)$, and $d S_{t}=\sigma\left(t, S_{t}\right) d M_{t}+\mu\left(t, S_{t}\right) d t$, we can apply Proposition 2.3 with $\left(X_{t}\right)_{t \in[0, T]}=\left(\left(S_{t}, M_{t}\right)\right)_{t \in[0, T]}$ and

$$
\begin{aligned}
L_{t} f\left(S_{t}, M_{t}\right) & =i_{t} \sigma\left(t, S_{t}\right) \partial_{1} f\left(S_{t}, M_{t}\right)+i_{t} \partial_{2} f\left(S_{t}, M_{t}\right) \\
+ & \frac{j_{t}}{\varphi\left(t, M_{t}\right)}\left(f\left(S_{t}+\varphi\left(t, M_{t}\right) \sigma\left(t, S_{t}\right), M_{t}+\varphi\left(t, M_{t}\right)\right)-f\left(S_{t}, M_{t}\right)\right),
\end{aligned}
$$

since the possible jump of $\left(M_{t}\right)_{t \in[0, T]}$ at time $t$ is $\varphi\left(t, M_{t}\right)$. Here $\partial_{1}$, resp. $\partial_{2}$, denotes the partial derivative with respect to the first, resp. second, variable. Hence

$$
\begin{aligned}
E\left[D _ { t } f \left(S_{T},\right.\right. & \left.\left.M_{T}\right) \mid \mathcal{F}_{t}\right] \\
= & i_{t} \sigma\left(t, S_{t}\right)\left(\partial_{1} P_{t, T} f\right)\left(\left(S_{t}, M_{t}\right)\right)+i_{t}\left(\partial_{2} P_{t, T} f\right)\left(\left(S_{t}, M_{t}\right)\right) \\
& +\frac{j_{t}}{\varphi\left(t, M_{t}\right)}\left(P_{t, T} f\right)\left(\left(S_{t}+\varphi\left(t, M_{t}\right) \sigma\left(t, S_{t}\right), M_{t}+\varphi\left(t, M_{t}\right)\right)\right) \\
& -\frac{j_{t}}{\varphi\left(t, M_{t}\right)}\left(P_{t, T} f\right)\left(\left(S_{t}, M_{t}\right)\right) .
\end{aligned}
$$

If $\left(M_{t}\right)_{t \in[0, T]}$ is an Azéma martingale $\left(\phi_{t}=\beta M_{t}\right.$ for $\left.t \in[0, T]\right)$, then $i_{t}=0$ $d P \times d t$ a.s.

\section{Computations of hedging strategies}

4.1. Market model. In this subsection we introduce the price process which will be considered in what follows. Let $\mu:[0, T] \rightarrow \mathbb{R}$ and $\sigma:[0, T] \rightarrow$ ] $0, \infty$ [ be deterministic bounded functions. Let $\left(r_{t}\right)_{t \in[0, T]}$ be a deterministic non-negative function which models a riskless asset, and let $\left(\psi_{t}\right)_{t \in[0, T]}$ be defined as

$$
\psi_{t}=\frac{r_{t}-\mu_{t}}{\sigma_{t} \alpha_{t}^{2}}, \quad t \in[0, T] .
$$

We assume that $1+\phi_{t} \psi_{t}>0$ for $t \in[0, T]$. If $\left(\phi_{t}\right)_{t \in[0, T]}$ is not deterministic this choice is still possible due to the boundedness of $\left(\phi_{t}\right)_{t \in[0, T]}$ or from [DP99, Th. 2.1(iii)]. Let $Q$ denote the probability defined by $E\left[\frac{d Q}{d P} \mid \mathcal{F}_{t}\right]=l_{t}$ for $t \in[0, T]$, where $d l_{t}=l_{t^{-}} \psi_{t} d M_{t}$ for $t \in[0, T]$, with $l_{0}=1$. From Proposition 2.4, 


$$
Z_{t}=M_{t}-\int_{0}^{t} \alpha_{s}^{2} \psi_{s} d s, \quad t \in[0, T],
$$

is a local martingale under $Q$ with angle bracket $d\langle Z, Z\rangle_{t}=\alpha_{t}^{2}\left(1+\phi_{t} \psi_{t}\right) d t$. If $\left(\phi_{t}\right)_{t \in[0, T]}$ is deterministic then $\left(Z_{t}\right)_{t \in[0, T]}$ is a true martingale under $Q$. This also holds if

$$
\left(\phi_{t}\right)_{t \in[0, T]}=\left(\beta M_{t}\right)_{t \in[0, T]},
$$

from the boundedness of $\left(M_{t}\right)_{t \in[0, T]}$. If $\left(\phi_{t}\right)_{t \in[0, T]}$ is deterministic, we may start from the data of $\left(\widetilde{\alpha}_{t}\right)_{t \in[0, T]}$, take $d[Z, Z]_{t}=\widetilde{\alpha}_{t}^{2} d t+\phi_{t} d Z_{t}$ and define $\left(\alpha_{t}\right)_{t \in[0, T]}$ by $\alpha_{t}^{2}=\widetilde{\alpha}_{t}^{2} /\left(1+\phi_{t} \psi_{t}\right)$ for $t \in[0, T]$. Let the price $\left(S_{t}\right)_{t \in[0, T]}$ of a risky asset satisfy the equation

$$
d S_{t}=\mu_{t} S_{t} d t+\sigma_{t} S_{t^{-}} d Z_{t}, \quad t \in[0, T], \quad S_{0}=1 .
$$

We have

$$
d S_{t}=r_{t} S_{t} d t+\sigma_{t} S_{t^{-}} d M_{t}, \quad t \in[0, T],
$$

and under $P,\left(S_{t} e^{-\int_{0}^{t} r_{s} d s}\right)_{t \in[0, T]}$ is a martingale, i.e. the market is arbitrage free. Let $\eta_{t}$ and $\zeta_{t}$ denote the number of units invested at time $t$ in the risky and riskless assets respectively. Thus the value $V_{t}$ of the portfolio at time $t$ is given by

$$
V_{t}=\zeta_{t} A_{t}+\eta_{t} S_{t}, \quad t \in[0, T]
$$

where

$$
d A_{t}=r_{t} A_{t} d t, \quad A_{0}=1, \quad t \in[0, T] .
$$

We assume that the portfolio is self-financing, i.e.

$$
d V_{t}=\zeta_{t} d A_{t}+\eta_{t} d S_{t}, \quad t \in[0, T],
$$

therefore

$$
d V_{t}=r_{t} V_{t} d t+\sigma_{t} \eta_{t} S_{t^{-}} d M_{t}, \quad t \in[0, T],
$$

and

$$
V_{T} e^{-\int_{0}^{T} r_{t} d t}=V_{0}+\int_{0}^{T} \sigma_{t} \eta_{t} S_{t^{-}} e^{-\int_{0}^{t} r_{s} d s} d M_{t},
$$

Suppose that we are required to find a portfolio $\left(\zeta_{t}, \eta_{t}\right)_{t \in[0, T]}$ which leads to a given value $V_{T}=F$. By the Clark-Ocone formula,

$$
F=E[F]+\int_{0}^{T} E\left[D_{t} F \mid \mathcal{F}_{t}\right] d M_{t},
$$

and comparing with (4.1.5) we obtain

$$
\begin{aligned}
V_{0} & =e^{-\int_{0}^{T} r_{s} d s} E[F], \\
\eta_{t} & =\sigma_{t}^{-1} S_{t}^{-1} E\left[D_{t} F \mid \mathcal{F}_{t}\right] e^{-\int_{t}^{T} r_{s} d s}, \quad t \in[0, T] .
\end{aligned}
$$


Next we consider different models with explicit computations of hedging strategies.

4.2. European options and deterministic structure. In this section we hedge European calls using the Clark formula. Let $\left(M_{t}\right)_{t \in[0, T]}$ be the martingale described in Section 2.2(a), with deterministic $\left(\phi_{t}\right)_{t \in[0, T]}$, i.e. $\left(M_{t}\right)_{t \in[0, T]}$ is alternatively Brownian or Poisson depending on the vanishing of $\left(\phi_{t}\right)_{t \in[0, T]}$. We assume that $1+\sigma_{t} \phi_{t}>0$ for all $t \in[0, T]$. We have

$$
S_{t}=\exp \left(\int_{0}^{t} \sigma_{s} \alpha_{s} i_{s} d B_{s}+\int_{0}^{t}\left(r_{s}-\phi_{s} \lambda_{s} \sigma_{s}-\frac{1}{2} i_{s} \sigma_{s}^{2} \alpha_{s}^{2}\right) d s\right) \prod_{k=1}^{N_{t}}\left(1+\sigma_{T_{k}} \phi_{T_{k}}\right)
$$

for $0 \leq t \leq T$, where $\left(T_{k}\right)_{k \geq 1}$ denotes the jump times of $\left(N_{t}\right)_{t \in \mathbb{R}_{+}}$. We will denote by $\left(S_{t, u}^{x}\right)_{u \in[t, T]}$ the process defined as

$$
d S_{t, u}^{x}=r_{t} S_{t, u}^{x} d u+\sigma_{u} S_{t, u^{-}}^{x} d M_{u}, \quad u \in[t, T], \quad S_{t, t}^{x}=x .
$$

We have

$$
\begin{aligned}
S_{t, T}^{x}= & x \exp \left(\int_{t}^{T} \sigma_{u} \alpha_{u} i_{u} d B_{u}+\int_{t}^{T}\left(r_{u}-\phi_{u} \lambda_{u} \sigma_{u}-\frac{1}{2} i_{u} \sigma_{u}^{2} \alpha_{u}^{2}\right) d u\right) \\
& \times \prod_{k=1+N_{t}}^{N_{T}}\left(1+\sigma_{T_{k}} \phi_{T_{k}}\right)
\end{aligned}
$$

for $0 \leq t \leq T$, with $S_{t}=S_{0, t}^{1}$ for $t \in[0, T]$.

Proposition 4.1. Assume that $\phi_{t} \geq 0$ for all $t \in[0, T]$. Then for $0 \leq$ $t \leq T$ we have

$$
\begin{aligned}
E\left[D_{t}\left(S_{T}-K\right)^{+} \mid \mathcal{F}_{t}\right]= & E\left[i_{t} \sigma_{t} S_{t, T}^{x} 1_{[K, \infty[}\left(S_{t, T}^{x}\right)\right. \\
& \left.+\frac{j_{t}}{\phi_{t}}\left(\sigma_{t} \phi_{t} S_{t, T}^{x}-\left(K-S_{t, T}^{x}\right)^{+}\right) 1_{\left[\frac{K}{1+\sigma_{t}}, \infty[\right.}\left(S_{t, T}^{x}\right)\right]_{x=S_{t}} .
\end{aligned}
$$

Proof. By Proposition 3.2, for any $F \in \mathcal{S}$ we have

$$
D_{t} F=D_{t}^{B} F+\frac{j_{t}}{\phi_{t}}\left(T_{t}^{\phi} F-F\right), \quad t \in[0, T] .
$$

We have $T_{t}^{\phi} S_{T}=\left(1+\sigma_{t} \phi_{t}\right) S_{T}$ for $t \in[0, T]$, and the chain rule $D^{B} f(F)=$ $f^{\prime}(F) D^{B} F$ holds for $F \in \mathcal{S}$ and $f \in \mathcal{C}_{\mathrm{b}}^{2}(\mathbb{R})$. Since $\mathcal{S}$ is an algebra for deterministic $\left(\phi_{t}\right)_{t \in[0, T]}$, we may approach $x \mapsto(x-K)^{+}$by polynomials on compact intervals and proceed e.g. as in [Øks96, pp. 5-13]. By dominated convergence, $\left(S_{T}-K\right)^{+} \in \operatorname{Dom}(D)$ and (4.2.1) becomes

$$
\left.D_{t}\left(S_{T}-K\right)^{+}=i_{t} \sigma_{t} S_{T} 1_{[K, \infty[}\left(S_{T}\right)+\frac{j_{t}}{\phi_{t}}\left(\left(1+\sigma_{t} \phi_{t}\right) S_{T}-K\right)^{+}-\left(S_{T}-K\right)^{+}\right)
$$


for $0 \leq t \leq T$. The Markov property of $\left(S_{t}\right)_{t \in[0, T]}$ implies

$$
E\left[D_{t}^{B}\left(S_{T}-K\right)^{+} \mid \mathcal{F}_{t}\right]=i_{t} \sigma_{t} E\left[S_{t, T}^{x} 1_{[K, \infty[}\left(S_{t, T}^{x}\right)\right]_{x=S_{t}},
$$

and

$$
\begin{aligned}
\frac{j_{t}}{\phi_{t}} E\left[\left(T_{t}^{\phi}\right.\right. & \left.\left.S_{T}-K\right)^{+}-\left(S_{T}-K\right)^{+} \mid \mathcal{F}_{t}\right] \\
= & \frac{j_{t}}{\phi_{t}} E\left[\left(\left(1+\sigma_{t} \phi_{t}\right) S_{t, T}^{x}-K\right)^{+}-\left(S_{t, T}^{x}-K\right)^{+}\right]_{x=S_{t}} \\
= & \frac{j_{t}}{\phi_{t}} E\left[\left(\left(1+\sigma_{t} \phi_{t}\right) S_{t, T}^{x}-K\right) 1_{\left[\frac{K}{1+\sigma_{t} \phi_{t}}, \infty[\right.}\left(S_{t, T}^{x}\right)\right]_{x=S_{t}} \\
& -\frac{j_{t}}{\phi_{t}} E\left[\left(S_{t, T}^{x}-K\right)^{+} 1_{[K, \infty[}\left(S_{t, T}^{x}\right)\right]_{x=S_{t}} \\
= & \frac{j_{t}}{\phi_{t}} E\left[\sigma_{t} \phi_{t} S_{t, T}^{x} 1_{\left[\frac{K}{1+\sigma_{t} \phi_{t}}, \infty[\right.}\left(S_{t, T}^{x}\right)+\left(S_{t, T}^{x}-K\right) 1_{\left[\frac{K}{1+\sigma_{t} \phi_{t}}, K\right]}\left(S_{t, T}^{x}\right)\right]_{x=S_{t}} \\
= & \frac{j_{t}}{\phi_{t}} E\left[\sigma_{t} \phi_{t} S_{t, T}^{x} 1_{\left[\frac{K}{1+\sigma_{t} \phi_{t}}, \infty[\right.}\left(S_{t, T}^{x}\right)-\left(K-S_{t, T}^{x}\right)^{+} 1_{\left[\frac{K}{1+\sigma_{t} \phi_{t}}, \infty\right]}\left(S_{t, T}^{x}\right)\right]_{x=S_{t}} \\
= & \frac{j_{t}}{\phi_{t}} E\left[\left(\sigma_{t} \phi_{t} S_{t, T}^{x}-\left(K-S_{t, T}^{x}\right)^{+}\right) 1_{\left[\frac{K}{1+\sigma_{t} \phi_{t}}, \infty[\right.}\left(S_{t, T}^{x}\right)\right]_{x=S_{t}} . \cdot-
\end{aligned}
$$

If $\left(\phi_{t}\right)_{t \in[0, T]}$ is not constrained to be positive then

$$
\begin{aligned}
E\left[D _ { t } \left(S_{T}\right.\right. & \left.-K)^{+} \mid \mathcal{F}_{t}\right]=i_{t} \sigma_{t} E\left[S_{t, T}^{x} 1_{[K, \infty[}\left(S_{t, T}^{x}\right)\right]_{x=S_{t}} \\
& +\frac{j_{t}}{\phi_{t}} E\left[\sigma_{t} \phi_{t} S_{t, T}^{x} 1_{\left[\frac{K}{1+\sigma_{t} \phi_{t}}, \infty[\right.}\left[S_{t, T}^{x}\right)+\left(S_{t, T}^{x}-K\right) 1_{\left[\frac{K}{1+\sigma_{t} \phi_{t}}, K\right]}\left(S_{t, T}^{x}\right)\right]_{x=S_{t}},
\end{aligned}
$$

with the convention $1_{[b, a]}=-1_{[a, b]}$ for $0 \leq a<b \leq T$. Proposition 4.1 can also be proved using Lemma 3.3 and the Itô formula (2.3.1). In the deterministic case, the semigroup $P_{t, T}$ can be explicitly computed. Let $\Gamma_{t, T}^{\sigma}=$ $\int_{t}^{T} i_{s} \alpha_{s}^{2} \sigma_{s}^{2} d s$ denote the variance of $\int_{t}^{T} i_{s} \alpha_{s} \sigma_{s} d B_{s}$ for $t \in[0, T]$, and let $\Gamma_{t, T}=\int_{t}^{T} \gamma_{s} d s, t \in[0, T]$, denote the intensity of $N_{T}-N_{t}$ under $Q$, where $\gamma_{t}=\lambda_{t}\left(1+\phi_{t} \psi_{t}\right)$ for $t \in[0, T]$.

Proposition 4.2. For $f \in \mathcal{C}_{\mathrm{b}}(\mathbb{R})$ we have

$$
\begin{aligned}
P_{t, T} f(x)= & \sum_{k=0}^{\infty} \frac{e^{-\Gamma_{t, T}}}{k !} \int_{-\infty}^{\infty} \nu_{t_{0}} \int_{[t, T]^{k}} \gamma_{t_{1}} \ldots \gamma_{t_{k}} \\
& \times f\left(x e^{-\Gamma_{t, T}^{\sigma} / 2+\left(\Gamma_{t, T}^{\sigma}\right)^{1 / 2} t_{0}-\int_{t}^{T} \phi_{s} \gamma_{s} \sigma_{s} d s} \prod_{i=1}^{k}\left(1+\sigma_{t_{i}} \phi_{t_{i}}\right)\right) d t_{1} \ldots d t_{k} d t_{0},
\end{aligned}
$$

where $\nu$ denotes the standard Gaussian density. 
Proof. We have $P_{t, T} f(x)=E\left[f\left(S_{T}\right) \mid S_{t}=x\right]=E\left[f\left(S_{t, T}^{x}\right)\right]$, and

$$
P_{t, T} f(x)=\sum_{k=0}^{\infty} E\left[f\left(S_{t, T}^{x}\right) \mid N_{T}-N_{t}=k\right] \exp \left(-\Gamma_{t, T}\right) \frac{\Gamma_{t, T}^{k}}{k !} .
$$

For $k \in \mathbb{N}$, since $\left(N_{\Gamma_{t, s}^{-1}}-N_{t}\right)_{s \in[t, T]}$ is a standard Poisson process, conditionally on $\left\{N_{T}-N_{t}=k\right\}$, the first $n$ jump times $\left(T_{1}, \ldots, T_{n}\right)$ of $\left(N_{s}\right)_{s \in[t, T]}$ have the law

$$
\frac{k !}{\left(\Gamma_{t, T}\right)^{k}} 1_{\left\{t<t_{1}<\ldots<t_{k}<T\right\}} \gamma_{t_{1}} \ldots \gamma_{t_{k}} d t_{1} \ldots d t_{k},
$$

and conditionally on $\left\{N_{T}-N_{t}=k\right\}$, the jump times $\left(\Gamma_{t, T_{1}}, \ldots, \Gamma_{t, T_{k}}\right)$ have a uniform law on $\left[0, \Gamma_{t, T}\right]^{k}$. We then use the identity in law between $S_{t, T}^{x}$ and

$$
x X_{t, T} \exp \left(-\int_{t}^{T} \phi_{s} \lambda_{s}\left(1+\phi_{s} \psi_{s}\right) \sigma_{s} d s\right) \prod_{k=1+N_{t}}^{N_{T}}\left(1+\sigma_{T_{k}} \phi_{T_{k}}\right),
$$

where

$$
X_{t, T}=\exp \left(-\Gamma_{t, T}^{\sigma} / 2+\left(\Gamma_{t, T}^{\sigma}\right)^{1 / 2} W\right),
$$

and $W$ a standard Gaussian random variable, independent of $\left(N_{t}\right)_{t \in[0, T]}$. This identity holds because $\left(B_{t}\right)_{t \in[0, T]}$ is a standard Brownian motion, independent of $\left(N_{t}\right)_{t \in[0, T]}$.

See Proposition 8 of [JP02] for a computation of

$$
E\left[\exp \left(-\int_{0}^{T} r_{s} d s\right)\left(S_{T}-K\right)^{+}\right]
$$

in terms of the classical Black-Scholes function

$$
\mathrm{BS}\left(x, T ; r, \sigma^{2} ; K\right)=E\left[e^{-r T}\left(x e^{r T-\sigma^{2} T / 2+\sigma W_{t}}-K\right)^{+}\right],
$$

where $W_{t}$ is a centered Gaussian random variable with variance $t$.

4.3. Asian options and deterministic structure. The price at time $t$ of such an option is

$$
E\left[e^{-\int_{t}^{T} r_{s} d s}\left(\frac{1}{T} \int_{0}^{T} S_{u} d u-K\right)^{+} \mid \mathcal{F}_{t}\right] .
$$

The next proposition gives us a replicating hedging strategy for Asian options in the case of a deterministic structure equation model. Following [LL96, p. 91], we define the auxiliary process

$$
Y_{t}=\frac{1}{S_{t}}\left(\frac{1}{T} \int_{0}^{t} S_{u} d u-K\right), \quad t \in[0, T] .
$$


Proposition 4.3. There exists a measurable function $\widetilde{C}$ on $\mathbb{R}_{+} \times \mathbb{R}$ such that $\widetilde{C}(t, \cdot)$ is $\mathcal{C}^{1}$ for all $t \in \mathbb{R}_{+}$, and

$$
S_{t} \widetilde{C}\left(t, Y_{t}\right)=E\left[\left(\frac{1}{T} \int_{0}^{T} S_{u} d u-K\right)^{+} \mid \mathcal{F}_{t}\right] .
$$

Moreover, the replicating portfolio for an Asian option with payoff

$$
\left(\frac{1}{T} \int_{0}^{T} S_{u} d u-K\right)^{+}
$$

is given by (4.1.3) and

$$
\begin{aligned}
& \eta_{t}=\frac{1}{\sigma_{t}} e^{-\int_{t}^{T} r_{s} d s}\left[\widetilde{C}\left(t, Y_{t}\right) \sigma_{t}\right. \\
& \left.+\left(1+\sigma_{t} \phi_{t}\right)\left(\frac{j_{t}}{\phi_{t}}\left(\widetilde{C}\left(t, \frac{Y_{t}}{1+\sigma_{t} \phi_{t}}\right)-\widetilde{C}\left(t, Y_{t}\right)\right)-i_{t} \sigma_{t} Y_{t} \partial_{2} \widetilde{C}\left(t, Y_{t}\right)\right)\right] .
\end{aligned}
$$

Proof. With the above notation, the price of the Asian option at time $t$ becomes

$$
E\left[e^{-\int_{t}^{T} r_{s} d s} S_{T}\left(Y_{T}\right)^{+} \mid \mathcal{F}_{t}\right]
$$

For $0 \leq s \leq t \leq T$, we have

$$
d\left(S_{t} Y_{t}\right)=\frac{1}{T} d\left(\int_{0}^{t} S_{u} d u-K\right)=\frac{S_{t}}{T} d t,
$$

hence

$$
\frac{S_{t} Y_{t}}{S_{s}}=Y_{s}+\frac{1}{T} \int_{s}^{t} \frac{S_{u}}{S_{s}} d u
$$

Let $H \in \mathcal{C}_{\mathrm{b}}^{2}(\mathbb{R})$. We have

$$
\begin{aligned}
E\left[H\left(S_{T} Y_{T}\right) \mid \mathcal{F}_{t}\right] & =E\left[H\left(S_{t} Y_{t}+\frac{1}{T} \int_{t}^{T} S_{u} d u\right) \mid \mathcal{F}_{t}\right] \\
& =E\left[H\left(x y+\frac{x}{T} \int_{t}^{T} \frac{S_{u}}{S_{t}} d u\right)\right]_{y=Y_{t}, x=S_{t}} .
\end{aligned}
$$

Let $C \in \mathcal{C}_{\mathrm{b}}^{2}\left(\mathbb{R}_{+} \times \mathbb{R}^{2}\right)$ be defined as

$$
C(t, x, y)=E\left[H\left(x y+\frac{x}{T} \int_{t}^{T} \frac{S_{u}}{S_{t}} d u\right)\right],
$$

i.e.

$$
C\left(t, S_{t}, Y_{t}\right)=E\left[H\left(S_{T} Y_{T}\right) \mid \mathcal{F}_{t}\right]
$$


When $H(x)=x^{+}$, since for any $t \in[0, T], S_{t}$ is positive and $\mathcal{F}_{t}$-measurable, and $S_{u} / S_{t}$ is independent of $\mathcal{F}_{t}$ for $u \geq t$, we have

$$
\begin{aligned}
E\left[H\left(S_{T} Y_{T}\right) \mid \mathcal{F}_{t}\right] & =E\left[S_{T}\left(Y_{T}\right)^{+} \mid \mathcal{F}_{t}\right]=S_{t} E\left[\left(\frac{S_{T}}{S_{t}} Y_{T}\right)^{+} \mid \mathcal{F}_{t}\right] \\
& =S_{t} E\left[\left(Y_{t}+\frac{1}{T} \int_{t}^{T} \frac{S_{u}}{S_{t}} d u\right)^{+} \mid \mathcal{F}_{t}\right] \\
& =S_{t} E\left[\left(y+\frac{1}{T} \int_{t}^{T} \frac{S_{u}}{S_{t}} d u\right)^{+} \mid\right]_{y=Y_{t}}=S_{t} \widetilde{C}\left(t, Y_{t}\right)
\end{aligned}
$$

with

$$
\widetilde{C}(t, y)=E\left[\left(y+\frac{1}{T} \int_{t}^{T} \frac{S_{u}}{S_{t}} d u\right)^{+}\right] .
$$

We now proceed as in [Bel99], which deals with the sum of a Brownian motion and a Poisson process. From the expression for $1 / S_{t}$ we have

$$
d\left(\frac{1}{S_{t}}\right)=\frac{1}{S_{t^{-}}}\left[\left(-r_{t}+\frac{\alpha_{t}^{2} \sigma_{t}^{2}}{1+\sigma_{t} \phi_{t}}\right) d t-\frac{\sigma_{t}}{1+\sigma_{t} \phi_{t}} d M_{t}\right],
$$

hence by $(2.3 .1)$,

$$
d Y_{t}=Y_{t}\left(-r_{t}+\frac{\alpha_{t}^{2} \sigma_{t}^{2}}{1+\sigma_{t} \phi_{t}}\right) d t+\frac{1}{T} d t-\frac{Y_{t^{-}} \sigma_{t}}{1+\sigma_{t} \phi_{t}} d M_{t} .
$$

Applying Lemma 3.3 we get

$$
\begin{aligned}
& E\left[D_{t} H\left(S_{T} Y_{T}\right) \mid \mathcal{F}_{t}\right]=L_{t} C\left(t, S_{t}, Y_{t}\right) \\
& =i_{t}\left(\sigma_{t} S_{t^{-}} \partial_{2} C\left(t, S_{t}, Y_{t}\right)-\frac{Y_{t} \sigma_{t}}{1+\sigma_{t} \phi_{t}} \partial_{3} C\left(t, S_{t}, Y_{t}\right)\right) \\
& \quad+\frac{j_{t}}{\phi_{t}}\left(C\left(t, S_{t^{-}}+\sigma_{t} S_{t^{-}}, Y_{t^{-}}-\frac{Y_{t} \sigma_{t}}{1+\sigma_{t} \phi_{t}}\right)-C\left(t, S_{t^{-}}, Y_{t^{-}}\right)\right) .
\end{aligned}
$$

Given a family $\left(H_{n}\right)_{n \in \mathbb{N}}$ of $\mathcal{C}_{\mathrm{b}}^{2}$ functions such that $\left|H_{n}(x)\right| \leq x^{+}$and $\left|H_{n}^{\prime}(x)\right|$ $\leq 2$ for $x \in \mathbb{R}$ and $n \in \mathbb{N}$, and converging pointwise to $x \mapsto x^{+}$, by dominated convergence (4.3.2) holds for $C(t, x, y)=x \widetilde{C}(t, y)$ and we obtain

$$
\begin{aligned}
E\left[D_{t}(\right. & \left.\left.\frac{1}{T} \int_{0}^{T} S_{u} d u-K\right)^{+} \mid \mathcal{F}_{t}\right] \\
= & i_{t} \widetilde{C}\left(t, Y_{t}\right) \sigma_{t} S_{t} \\
& +S_{t}\left(\frac{j_{t}}{\phi_{t}}\left(\widetilde{C}\left(t, \frac{Y_{t}}{1+\sigma_{t} \phi_{t}}\right)-\widetilde{C}\left(t, Y_{t}\right)\right)-i_{t} \sigma_{t} Y_{t} \partial_{2} \widetilde{C}\left(t, Y_{t}\right)\right) \\
& +S_{t} \sigma_{t} \phi_{t}\left(\frac{j_{t}}{\phi_{t}}\left(\widetilde{C}\left(t, \frac{Y_{t}}{1+\sigma_{t} \phi_{t}}\right)-\widetilde{C}\left(t, Y_{t}\right)\right)-i_{t} \sigma_{t} Y_{t} \partial_{2} \widetilde{C}\left(t, Y_{t}\right)\right)
\end{aligned}
$$


As a particular case we consider the Brownian motion model, i.e. $\phi_{t}=0$ for all $t \in[0, T]$, so $i_{t}=1, j_{t}=0$ for all $t \in[0, T]$. In this case we have

$$
\begin{aligned}
\eta_{t} & =e^{-\int_{t}^{T} r_{s} d s}\left(-Y_{t} \partial_{2} \widetilde{C}\left(t, Y_{t}\right)+\widetilde{C}\left(t, Y_{t}\right)\right) \\
& =e^{-\int_{t}^{T} r_{s} d s}\left(\left.S_{t} \frac{\partial}{\partial x} \widetilde{C}\left(t, \frac{1}{x}\left(\frac{1}{T} \int_{0}^{t} S_{u} d u-K\right)\right)\right|_{x=S_{t}}+\widetilde{C}\left(t, Y_{t}\right)\right) \\
& =\left.\frac{\partial}{\partial x}\left(x e^{-\int_{t}^{T} r_{s} d s} \widetilde{C}\left(t, \frac{1}{x}\left(\frac{1}{T} \int_{0}^{t} S_{u} d u-K\right)\right)\right)\right|_{x=S_{t}}, \quad t \in[0, T],
\end{aligned}
$$

which can be denoted informally as a partial derivative with respect to $S_{t}$.

4.4. European options and Azéma martingales. Let $-2 \leq \beta<0$, and let $\left(M_{t}\right)_{t \in[0, T]}$ be the unique solution of the structure equation

$$
d[M, M]_{t}=d t+\beta M_{t^{-}} d M_{t}, \quad t \in[0, T] .
$$

This process has the chaos representation property, hence the results of Section 3 apply. This allows us to obtain an explicit hedging formula for the model of [DP99]. We use the convention $1_{[b, a]}=-1_{[a, b]}$ for $0 \leq a<b \leq T$.

Proposition 4.4. We have

$$
\begin{aligned}
E\left[D_{t}\left(S_{T}-K\right)^{+} \mid \mathcal{F}_{t}\right] & \\
= & \frac{1}{\beta M_{t}} E\left[\sigma_{t} \beta\left(y+M_{T}-M_{t}\right) S_{t, T}^{x} 1_{\left[\frac{K}{1+\sigma_{t} \beta\left(y+M_{T}-M_{t}\right)}, \infty[\right.}\left(S_{t, T}^{x}\right)\right. \\
& \left.+\left(S_{t, T}^{x}-K\right) 1_{\left[\frac{K}{1+\sigma_{t} \beta\left(y+M_{T}-M_{t}\right)}, K\right]}\left(S_{t, T}^{x}\right)\right]_{x=S_{t}}^{y=M_{t}} .
\end{aligned}
$$

Proof. Let $\left(X_{t}\right)_{t \in[0, T]}=\left(\left(S_{t}, M_{t}\right)\right)_{t \in[0, T]},\left(R_{t}\right)_{t \in[0, T]}=\left(\left(r_{t} S_{t}, 0\right)\right)_{t \in[0, T]}$, $\left(K_{t}\right)_{t \in[0, T]}=\left(\left(\sigma_{t} S_{t}, 1\right)\right)_{t \in[0, T]}$, and $X_{0}=(1,0)$. By Lemma 3.3, for $f \in$ $\mathcal{C}_{\mathrm{b}}^{2}\left(\mathbb{R}^{2}\right)$ we have

$$
\begin{aligned}
E\left[D_{t} f\left(X_{T}\right) \mid \mathcal{F}_{t}\right]=\left(L_{t}\left(P_{t, T} f\right)\right)\left(X_{t}\right) & \\
= & \frac{1}{\beta M_{t}}\left(\left(P_{t, T} f\right)\left(X_{t}+\beta M_{t} K_{t}\right)-\left(P_{t, T} f\right)\left(X_{t}\right)\right) \\
= & \frac{1}{\beta M_{t}}\left(\left(P_{t, T} f\right)\left(\left(1+\beta M_{t} \sigma_{t}\right) S_{t},(1+\beta) M_{t}\right)-\left(P_{t, T} f\right)\left(S_{t}, M_{t}\right)\right) \\
= & \frac{1}{\beta M_{t}} E\left[f \left(\left(1+\sigma_{t} \beta\left(y+M_{T}-M_{t}\right)\right) S_{t, T}^{x},\right.\right. \\
& \left.\left.\quad\left(1+\beta\left(y+M_{T}-M_{t}\right)\right)\right)\right]_{x=S_{t}}^{y=M_{t}} \\
& \quad \frac{1}{\beta M_{t}} E\left[f\left(S_{t, T}^{x}, y+M_{T}-M_{t}\right)\right]_{x=S_{t}}^{y=M_{t} .}
\end{aligned}
$$


In particular if $f(x, y)=f(x)$ depends only on the first variable we have

$$
\begin{aligned}
E\left[D_{t} f\left(S_{T}\right)\right. & \left.\mid \mathcal{F}_{t}\right] \\
& =\frac{1}{\beta M_{t}} E\left[f\left(\left(1+\sigma_{t} \beta\left(y+M_{T}-M_{t}\right)\right) S_{t, T}^{x}\right)-f\left(S_{t, T}^{x}\right)\right]_{x=S_{t}}^{y=M_{t}} .
\end{aligned}
$$

Approaching the function $x \mapsto(x-K)^{+}$with a sequence $\left(f_{n}\right)_{n \in \mathbb{N}}$ of $\mathcal{C}_{\mathrm{b}}^{2}$ functions converging pointwise with $\left|f_{n}(x)\right| \leq(x-K)^{+}$and $\left|f_{n}^{\prime}(x)\right| \leq 2$ for $x \in \mathbb{R}$ and $n \in \mathbb{N}$, we obtain

$$
\begin{aligned}
& E\left[D_{t}\left(S_{T}-K\right)^{+} \mid \mathcal{F}_{t}\right] \\
= & \frac{1}{\beta M_{t}} E\left[\left(\left(1+\sigma_{t} \beta\left(y+M_{T}-M_{t}\right)\right) S_{t, T}^{x}-K\right)^{+}-\left(S_{t, T}^{x}-K\right)^{+}\right]_{x=S_{t}}^{y=M_{t}} \\
= & \frac{1}{\beta M_{t}} E\left[\left(\left(1+\sigma_{t} \beta\left(y+M_{T}-M_{t}\right)\right) S_{t, T}^{x}-K\right) 1_{\left[\frac{K}{1+\sigma_{t} \beta\left(y+M_{T}-M_{t}\right)}, \infty[\right.}\left(S_{t, T}^{x}\right)\right. \\
& \left.-\left(S_{t, T}^{x}-K\right) 1_{[K, \infty[}\left(S_{t, T}^{x}\right)\right]_{x=S_{t}}^{y=M_{t}} \\
= & \frac{1}{\beta M_{t}} E\left[\sigma_{t} \beta\left(y+M_{T}-M_{t}\right) S_{t, T}^{x} 1_{\left[\frac{K}{1+\sigma_{t} \beta\left(y+M_{T}-M_{t}\right)}, \infty[\right.}\left(S_{t, T}^{x}\right)\right. \\
& \left.+\left(S_{t, T}^{x}-K\right) 1_{\left[\frac{K}{1+\sigma_{t} \beta\left(y+M_{T}-M_{t}\right)}, K\right]}\left(S_{t, T}^{x}\right)\right]_{x=S_{t}}^{y=M_{t}} .
\end{aligned}
$$

4.5. Lookback options. Hedging strategies for Lookback options have been computed in [Ber98] using the Clark-Ocone formula. In this section we show that classical martingale methods also apply in this case. We assume that $\left(M_{t}\right)_{t \in[0, T]}=\left(B_{t}\right)_{t \in[0, T]}$ is a standard Brownian motion, i.e. $\alpha_{t}=1$ and $\phi_{t}=0$ for every $t \in[0, T]$, and take $r_{t}=r \geq 0$ and $\sigma_{t}=\sigma \geq 0$ for every $t \in[0, T]$. Under the risk-free probability $P$ the asset price $\left(S_{t}\right)_{t \in[0, T]}$ has the dynamics

$$
d S_{t}=r S_{t} d t+\sigma S_{t} d B_{t}, \quad t \in[0, T]
$$

so (4.1.5) becomes

$$
V_{T} e^{-r T}=V_{0}+\int_{0}^{T} \sigma \eta_{t} S_{t} e^{-r t} d B_{t}, \quad t \in[0, T] .
$$

Let $m_{s}^{t}=\inf _{u \in[s, t]} S_{u}$ and $M_{s}^{t}=\sup _{u \in[s, t]} S_{u}$ for $0 \leq s \leq t \leq T$, and let $\mathcal{M}_{s}^{t}$ be either $m_{s}^{t}$ or $M_{s}^{t}$. In the Lookback option case the payoff $H\left(S_{T}, \mathcal{M}_{0}^{T}\right)$ depends not only on the price of the underlying asset at maturity but also on all prices of the asset from the initial time to maturity. Let $\mathrm{Look}_{t}$ be the price of the Lookback option given by

$$
\operatorname{Look}_{t}=e^{-r(T-t)} E\left[H\left(S_{T}, \mathcal{M}_{0}^{T}\right) \mid \mathcal{F}_{t}\right], \quad H \in \mathcal{C}_{\mathrm{b}}^{2}\left(\mathbb{R}^{2}\right), t \in[0, T] .
$$

Proposition 4.5. There exists a $\mathcal{C}^{1}$ function $f$ such that

$$
f\left(S_{t}, \mathcal{M}_{0}^{t}, t\right)=E\left[H\left(S_{T}, \mathcal{M}_{0}^{T}\right) \mid \mathcal{F}_{t}\right], \quad 0 \leq t \leq T .
$$


The replicating portfolio of a Lookback option with payoff $H\left(S_{T}, \mathcal{M}_{0}^{T}\right)$ and price $\operatorname{Look}_{t}=f\left(S_{t}, \mathcal{M}_{0}^{t}, t\right)$ at time $t$ is given by (4.1.3), and

$$
\eta_{t}=e^{-r(T-t)} \partial_{1} f\left(S_{t}, \mathcal{M}_{0}^{t}, t\right), \quad t \in[0, T] .
$$

Proof. It suffices to deal with the case $\mathcal{M}_{s}^{t}=m_{s}^{t}$. The existence of $f$ follows from the Markov property, more precisely

$$
f(x, y, t)=E\left[H\left(S_{t, T}^{x}, y \wedge \mathcal{M}_{t}^{T}\right)\right]
$$

Applying the change of variable formula, for $t \in[0, T]$ we have

$$
\begin{aligned}
d f\left(S_{t}, \mathcal{M}_{0}^{t}, t\right)= & {\left[\partial_{3} f+r S_{t} \partial_{1} f+\frac{1}{2} \sigma^{2} S_{t}^{2} \partial_{1}^{2} f\right]\left(S_{t}, \mathcal{M}_{0}^{t}, t\right) d t } \\
& +\partial_{2} f\left(S_{t}, \mathcal{M}_{0}^{t}, t\right) d \mathcal{M}_{0}^{t}+\sigma S_{t} \partial_{1} f\left(S_{t}, \mathcal{M}_{0}^{t}, t\right) d B_{t} .
\end{aligned}
$$

Since $\left(E\left[H\left(S_{T}, \mathcal{M}_{0}^{T}\right) \mid \mathcal{F}_{t}\right]\right)_{t \in[0, T]}$ is a $P$-martingale, we have

$$
d f\left(S_{t}, \mathcal{M}_{0}^{t}, t\right)=\sigma S_{t} \partial_{1} f\left(S_{t}, \mathcal{M}_{0}^{t}, t\right) d B_{t}, \quad t \in[0, T] .
$$

Then

$$
e^{-r T} F=e^{-r T} E[F]+\left.\int_{0}^{T} e^{-r T} \sigma S_{t} \frac{\partial}{\partial x} f\left(x, \mathcal{M}_{0}^{t}, t\right)\right|_{x=S_{t}} d B_{t}, \quad t \in[0, T],
$$

which shows (4.5.1).

It is stated in Bermin [Ber98] that we should have

$$
\int_{0}^{t} \partial_{2} f\left(S_{s}, \mathcal{M}_{0}^{s}, s\right) d \mathcal{M}_{0}^{s}=0
$$

for the delta hedging method to work. We showed in Proposition 4.5 that the delta hedging approach can be applied without having to verify (4.5.2), since $\left(\mathcal{M}_{0}^{t}\right)_{t \in[0, T]}$ is a monotone process with finite variation.

Relation (4.5.1) can be written informally as

$$
\eta_{t}=\frac{\partial}{\partial S_{t}} \operatorname{Look}_{t}, \quad t \in[0, T]
$$

Let

$$
d_{t}^{T}(y)=\frac{\log \frac{S_{t}}{y}+\left(r+\frac{1}{2} \sigma^{2}\right)(T-t)}{\sigma \sqrt{T-t}}, \quad \mathcal{N}(y)=\frac{1}{\sqrt{2 \pi}} \int_{-\infty}^{y} e^{-\frac{1}{2} u^{2}} d u .
$$

A standard Lookback call option is the right to buy the underlying asset at the historically lowest price. In this case the strike is $m_{0}^{T}$ and the payoff is

$$
G=S_{T}-m_{0}^{T}
$$

From [DJ98, Prop. 4, p. 271], the price Look $_{t}$ at time $t$ is given by

$$
\begin{aligned}
\operatorname{Look}_{t} & =E_{Q}\left[e^{-r(T-t)}\left(S_{T}-m_{0}^{T}\right) \mid \mathcal{F}_{t}\right] \\
& =S_{t} \mathcal{N}\left(d_{t}^{T}\left(m_{0}^{t}\right)\right)-e^{-r(T-t)} m_{0}^{t} \mathcal{N}\left(d_{t}^{T}\left(m_{0}^{t}\right)-\sigma \sqrt{T-t}\right)
\end{aligned}
$$




$$
\begin{aligned}
& +e^{-r(T-t)} \frac{S_{t} \sigma^{2}}{2 r}\left[\left(\frac{S_{t}}{m_{0}^{t}}\right)^{-2 r / \sigma^{2}} \mathcal{N}\left(-d_{t}^{T}\left(m_{0}^{t}\right)+\frac{2 r \sqrt{T-t}}{\sigma}\right)\right. \\
& \left.-e^{r(T-t)} \mathcal{N}\left(-d_{t}^{T}\left(m_{0}^{t}\right)\right)\right] .
\end{aligned}
$$

In the following proposition we recover the result of [Ber98, §2.6.1, p. 29], using the delta hedging approach instead of the Clark formula, as an application of Proposition 4.5.

Proposition 4.6. The hedging strategy for a standard Lookback call option is given by

$$
\begin{aligned}
\eta_{t}= & \mathcal{N}\left(d_{t}^{T}\left(m_{0}^{t}\right)\right)-\frac{\sigma^{2}}{2 r} \mathcal{N}\left(-d_{t}^{T}\left(m_{0}^{t}\right)\right) \\
& +e^{-r(T-t)}\left(\frac{S_{t}}{m_{0}^{t}}\right)^{-2 r / \sigma^{2}}\left(\frac{\sigma^{2}}{2 r}-1\right) \mathcal{N}\left(-d_{t}^{T}\left(m_{0}^{t}\right)+\frac{2 r \sqrt{T-t}}{\sigma}\right)
\end{aligned}
$$

Proof. We need to compute the following derivatives:

$$
\begin{aligned}
\frac{\partial}{\partial S_{t}} & \left(\mathcal{N}\left(d_{t}^{T}\left(m_{0}^{t}\right)\right)\right) \\
& =\frac{\partial}{\partial S_{t}}\left(\frac{1}{\sqrt{2 \pi}} \int_{-\infty}^{d_{t}^{T}\left(m_{0}^{t}\right)} e^{-\frac{1}{2} u^{2}} d u\right) \\
& =\frac{1}{\sqrt{2 \pi}}\left[\frac{\partial}{\partial S_{t}}\left(d_{t}^{T}\left(m_{0}^{t}\right)\right)\right] \exp \left(-\frac{1}{2}\left(d_{t}^{T}\left(m_{0}^{t}\right)\right)^{2}\right) \\
& =\frac{1}{\sqrt{2 \pi}}\left[\frac{\partial}{\partial S_{t}}\left(\frac{\log \frac{S_{t}}{m_{0}^{t}}+\left(r+\frac{1}{2} \sigma^{2}\right)(T-t)}{\sigma \sqrt{T-t}}\right)\right] \exp \left(-\frac{1}{2}\left(d_{t}^{T}\left(m_{0}^{t}\right)\right)^{2}\right) \\
& =\frac{1}{S_{t} \sigma \sqrt{2 \pi(T-t)}} \exp \left(-\frac{1}{2}\left(d_{t}^{T}\left(m_{0}^{t}\right)\right)^{2}\right)
\end{aligned}
$$

and

$$
\begin{aligned}
\frac{\partial}{\partial S_{t}}\left(\mathcal { N } \left(d_{t}^{T}\left(m_{0}^{t}\right)-\right.\right. & \sigma \sqrt{T-t})) \\
& =\frac{1}{S_{t} \sigma \sqrt{2 \pi(T-t)}} \exp \left(-\frac{1}{2}\left(d_{t}^{T}\left(m_{0}^{t}\right)-\sigma \sqrt{T-t}\right)^{2}\right) .
\end{aligned}
$$

Similarly we have

$$
\begin{aligned}
\frac{\partial}{\partial S_{t}}(\mathcal{N}(- & \left.\left.d_{t}^{T}\left(m_{0}^{t}\right)+\frac{2 r \sqrt{T-t}}{\sigma}\right)\right) \\
& =-\frac{1}{S_{t} \sigma \sqrt{2 \pi(T-t)}} \exp \left(-\frac{1}{2}\left(-d_{t}^{T}\left(m_{0}^{t}\right)+\frac{2 r \sqrt{T-t}}{\sigma}\right)^{2}\right),
\end{aligned}
$$


and

$$
\frac{\partial}{\partial S_{t}}\left(\mathcal{N}\left(-d_{t}^{T}\left(m_{0}^{t}\right)\right)\right)=-\frac{1}{S_{t} \sigma \sqrt{2 \pi(T-t)}} \exp \left(-\frac{1}{2}\left(d_{t}^{T}\left(m_{0}^{t}\right)\right)^{2}\right) .
$$

Finally,

$$
\frac{\partial}{\partial S_{t}}\left(\frac{S_{t}}{m_{0}^{t}}\right)^{-2 r / \sigma^{2}}=\frac{-2 r}{m_{0}^{t} \sigma^{2}}\left(\frac{S_{t}}{m_{0}^{t}}\right)^{-2 r / \sigma^{2}-1} .
$$

The above expressions can be combined to compute the derivative of Look $_{t}$ in (4.5.3), and to obtain

$$
\begin{aligned}
& \eta_{t}=\mathcal{N}\left(d_{t}^{T}\left(m_{0}^{t}\right)\right)+\frac{1}{\sigma \sqrt{2 \pi(T-t)}} \exp \left(-\frac{1}{2}\left(d_{t}^{T}\left(m_{0}^{t}\right)\right)^{2}\right) \\
& -e^{-r(T-t)} m_{0}^{t} \frac{1}{S_{t} \sigma \sqrt{2 \pi(T-t)}} \exp \left(-\frac{1}{2}\left(d_{t}^{T}\left(m_{0}^{t}\right)-\sigma \sqrt{T-t}\right)^{2}\right) \\
& +e^{-r(T-t)} \frac{\sigma^{2}}{2 r}\left[\left(\frac{S_{t}}{m_{0}^{t}}\right)^{-2 r / \sigma^{2}} \mathcal{N}\left(-d_{t}^{T}\left(m_{0}^{t}\right)+\frac{2 r \sqrt{T-t}}{\sigma}\right)\right. \\
& \left.-e^{r(T-t)} \mathcal{N}\left(-d_{t}^{T}\left(m_{0}^{t}\right)\right)\right] \\
& +e^{-r(T-t)} \frac{S_{t} \sigma^{2}}{2 r}\left[\frac{-2 r}{m_{0}^{t} \sigma^{2}}\left(\frac{S_{t}}{m_{0}^{t}}\right)^{-2 r / \sigma^{2}-1} \mathcal{N}\left(-d_{t}^{T}\left(m_{0}^{t}\right)+\frac{2 r \sqrt{T-t}}{\sigma}\right)\right. \\
& -\left(\frac{S_{t}}{m_{0}^{t}}\right)^{-2 r / \sigma^{2}} \frac{1}{S_{t} \sigma \sqrt{2 \pi(T-t)}} \exp \left(-\frac{1}{2}\left(-d_{t}^{T}\left(m_{0}^{t}\right)+\frac{2 r \sqrt{T-t}}{\sigma}\right)^{2}\right) \\
& \left.+e^{r(T-t)} \frac{1}{S_{t} \sigma \sqrt{2 \pi(T-t)}} \exp \left(-\frac{1}{2}\left(d_{t}^{T}\left(m_{0}^{t}\right)\right)^{2}\right)\right] \\
& =\mathcal{N}\left(-d_{t}^{T}\left(m_{0}^{t}\right)+\frac{2 r \sqrt{T-t}}{\sigma}\right)\left[e^{-r(T-t)} \frac{\sigma^{2}}{2 r}\left(\frac{S_{t}}{m_{0}^{t}}\right)^{-2 r / \sigma^{2}}+e^{-r(T-t)} \frac{S_{t} \sigma^{2}}{2 r}\right. \\
& \left.\times \frac{-2 r}{m_{0}^{t} \sigma^{2}}\left(\frac{S_{t}}{m_{0}^{t}}\right)^{-2 r / \sigma^{2}-1}\right]+\mathcal{N}\left(d_{t}^{T}\left(m_{0}^{t}\right)\right) \\
& -\mathcal{N}\left(-d_{t}^{T}\left(m_{0}^{t}\right)\right)\left[-e^{-r(T-t)} \frac{\sigma^{2}}{2 r} \cdot e^{r(T-t)}\right] \\
& +\frac{1}{\sigma \sqrt{2 \pi(T-t)}}\left\{\exp \left(-\frac{1}{2}\left(d_{t}^{T}\left(m_{0}^{t}\right)\right)^{2}\right)\left[1+e^{-r(T-t)} \frac{S_{t} \sigma^{2}}{2 r} \cdot \frac{e^{r(T-t)}}{S_{t}}\right]\right. \\
& -e^{-r(T-t)}\left[\frac{m_{0}^{t}}{S_{t}} \exp \left(-\frac{1}{2}\left(d_{t}^{T}\left(m_{0}^{t}\right)-\sigma \sqrt{T-t}\right)^{2}\right)\right. \\
& \left.\left.+\frac{\sigma^{2}}{2 r}\left(\frac{S_{t}}{m_{0}^{t}}\right)^{-2 r / \sigma^{2}} \exp \left(-\frac{1}{2}\left(-d_{t}^{T}\left(m_{0}^{t}\right)+\frac{2 r \sqrt{T-t}}{\sigma}\right)^{2}\right)\right]\right\}
\end{aligned}
$$




$$
\begin{aligned}
& =e^{-r(T-t)}\left(\frac{S_{t}}{m_{0}^{t}}\right)^{-2 r / \sigma^{2}}\left(\frac{\sigma^{2}}{2 r}-1\right) \mathcal{N}\left(-d_{t}^{T}\left(m_{0}^{t}\right)+\frac{2 r \sqrt{T-t}}{\sigma}\right) \\
& +\mathcal{N}\left(d_{t}^{T}\left(m_{0}^{t}\right)\right)-\frac{\sigma^{2}}{2 r} \mathcal{N}\left(-d_{t}^{T}\left(m_{0}^{t}\right)\right) \\
& +\frac{1}{\sigma \sqrt{2 \pi(T-t)}}\left\{\left(1+\frac{\sigma^{2}}{2 r}\right) \exp \left(-\frac{1}{2} d_{t}^{T}\left(m_{0}^{t}\right)^{2}\right)\right. \\
& -e^{-r(T-t)}\left[\frac{m_{0}^{t}}{S_{t}} \exp \left(-\frac{1}{2}\left(d_{t}^{T}\left(m_{0}^{t}\right)-\sigma \sqrt{T-t}\right)^{2}\right)\right. \\
& \left.\left.+\frac{\sigma^{2}}{2 r}\left(\frac{S_{t}}{m_{0}^{t}}\right)^{-2 r / \sigma^{2}} \exp \left(-\frac{1}{2}\left(-d_{t}^{T}\left(m_{0}^{t}\right)+\frac{2 r \sqrt{T-t}}{\sigma}\right)^{2}\right)\right]\right\} .
\end{aligned}
$$

To obtain (4.5.4), it is sufficient to show that

$$
\begin{aligned}
0= & \left(1+\frac{\sigma^{2}}{2 r}\right) \exp \left(-\frac{1}{2} d_{t}^{T}\left(m_{0}^{t}\right)^{2}\right) \\
& -e^{-r(T-t)}\left[\frac{m_{0}^{t}}{S_{t}} \exp \left(-\frac{1}{2}\left(d_{t}^{T}\left(m_{0}^{t}\right)-\sigma \sqrt{T-t}\right)^{2}\right)\right. \\
& \left.+\frac{\sigma^{2}}{2 r}\left(\frac{S_{t}}{m_{0}^{t}}\right)^{-2 r / \sigma^{2}} \exp \left(-\frac{1}{2}\left(-d_{t}^{T}\left(m_{0}^{t}\right)+\frac{2 r \sqrt{T-t}}{\sigma}\right)^{2}\right)\right] .
\end{aligned}
$$

To see this, one can observe that

$$
\begin{aligned}
\exp (- & \left.\frac{1}{2}\left(d_{t}^{T}(y)-\sigma \sqrt{T-t}\right)^{2}\right) \\
= & \exp \left(-\frac{1}{2}\left[\left(d_{t}^{T}(y)\right)^{2}+\sigma^{2}(T-t)-2 d_{t}^{T}(y) \sigma \sqrt{T-t}\right]\right) \\
= & \exp \left(-\frac{1}{2}\left(d_{t}^{T}(y)\right)^{2}\right) \\
& \times \exp \left(-\frac{1}{2}\left[\sigma^{2}(T-t)-2\left(\log \frac{S_{t}}{y}+\left(r+\frac{1}{2} \sigma^{2}\right)(T-t)\right)\right]\right) \\
= & \exp \left(-\frac{1}{2}\left[-2 \log \frac{S_{t}}{y}-2 r(T-t)\right]-\frac{1}{2}\left(d_{t}^{T}(y)\right)^{2}\right) \\
= & e^{r(T-t)} \frac{S_{t}}{y} \exp \left(-\frac{1}{2}\left(d_{t}^{T}(y)\right)^{2}\right)
\end{aligned}
$$

and

$$
\begin{aligned}
\exp \left(-\frac{1}{2}\left(-d_{t}^{T}(y)+2 r \sqrt{T-t} / \sigma\right)^{2}\right) & \\
& =\exp \left(-\frac{1}{2}\left(d_{t}^{T}(y)\right)^{2}-\frac{1}{2}\left[\frac{4 r^{2}}{\sigma^{2}}(T-t)-\frac{4 r}{\sigma} d_{t}^{T}(y) \sqrt{T-t}\right]\right)
\end{aligned}
$$




$$
\begin{aligned}
= & \exp \left(-\frac{1}{2}\left(d_{t}^{T}(y)\right)^{2}\right) \\
& \times \exp \left(-\frac{1}{2}\left[\frac{4 r^{2}}{\sigma^{2}}(T-t)-\frac{4 r}{\sigma^{2}}\left(\log \frac{S_{t}}{y}+\left(r+\frac{1}{2} \sigma^{2}\right)(T-t)\right)\right]\right) \\
= & \exp \left(-\frac{1}{2}\left(d_{t}^{T}(y)\right)^{2}\right) \\
& \times \exp \left(\frac{-2 r^{2}}{\sigma^{2}}(T-t)+\frac{2 r}{\sigma^{2}} \log \frac{S_{t}}{y}+\frac{2 r^{2}}{\sigma^{2}}(T-t)+r(T-t)\right) \\
= & e^{r(T-t)\left(\frac{S_{t}}{y}\right)^{2 r / \sigma^{2}} \exp \left(-\frac{1}{2}\left(d_{t}^{T}(y)\right)^{2}\right) .}
\end{aligned}
$$

Similar calculations using (4.5.1) are possible for other Lookback options, such as options on extrema and partial Lookback options (cf. [Kha02]).

Acknowledgments. We thank the referee for a careful reading of this paper, and E. Bichot for contributing to the simulation graphs. The results of this paper were presented in part at the AMS-Scandinavian meeting, Odense, 2000.

\section{References}

[AOPU00] K. Aase, B. Øksendal, N. Privault, and J. Ubøe, White noise generalizations of the Clark-Haussmann-Ocone theorem with application to mathematical finance, Finance Stoch. 4 (2000), 465-496.

[Bel99] N. Bellamy, Evaluation et couverture dans un marché dirigé par des processus discontinus, thèse, Univ. d'Evry, 1999.

[Ber98] H. Bermin, Essays on Lookback options: a Malliavin calculus approach, $\mathrm{PhD}$ thesis, Lund Univ., 1998.

[Cla70] J. M. C. Clark, The representation of functionals of Brownian motion by stochastic integrals, Ann. Math. Statist. 41 (1970), 1281-1295.

[DJ98] R.-A. Dana et M. Jeanblanc, Marchés financiers en temps continu, Economica, Paris, 1998.

[DP99] M. Dritschel and P. Protter, Complete markets with discontinuous security price, Finance Stoch. 3 (1999), 203-214.

[É90] M. Émery, On the Azéma martingales, in: Séminaire de Probabilités XXIII, Lecture Notes in Math. 1372, Springer, 1990, 66-87.

[JP02] M. Jeanblanc and N. Privault, A complete market model with Poisson and Brownian components, in: R. Dalang et al. (eds.), Seminar on Stochastic Analysis, Random Fields and Applications (Ascona, 1999), Progr. Probab., Birkhäuser, Basel, 2002, 189-204.

[Kha02] Y. El-Khatib, Contributions to the study of discontinuous markets via the Malliavin calculus, thèse, Univ. de La Rochelle, 2002. 
[KO91] I. Karatzas and D. L. Ocone, A generalized Clark representation formula with application to optimal portfolios, Stochastics Stochastics Rep. 34 (1991), 187-230.

[LL96] D. Lamberton and B. Lapeyre, Introduction to Stochastic Calculus Applied to Finance, Chapman \& Hall, London, 1996.

[Mey89] P.-A. Meyer, Construction de solutions d' "équations de structure", in: Séminaire de Probabilités XXIII, Lecture Notes in Math. 1372, Springer, Berlin, 1989, 142-145.

[Øks96] B. Øksendal, An introduction to Malliavin calculus with applications to economics, working paper no. 3, Inst. Finance and Management Sci., Norwegian School of Economics and Business Administration, 1996.

[Pha00] A. Phan, Contributions à l'étude des équations de structure markoviennes, thèse, Univ. de Strasbourg, 2000.

[Pri96] N. Privault, On the independence of multiple stochastic integrals with respect to a class of martingales, C. R. Acad. Sci. Paris Sér. I Math. 323 (1996), 515-520.

[Pro90] P. Protter, Stochastic Integration and Differential Equations. A New Approach, Springer, Berlin, 1990.

[Pro01] -, A partial introduction to financial asset pricing theory, Stochastic Process. Appl. 91 (2001), 169-203.

[PSV00] N. Privault, J. L. Solé, and J. Vives, Chaotic Kabanov formula for the Azéma martingales, Bernoulli 6 (2000), 633-651.

[Tav99] G. Taviot, Martingales et équations de structure : étude géométrique, thèse, Univ. de Strasbourg, 1999.

[Yor76] M. Yor, Sur les intégrales stochastiques optionnelles et une suite remarquable de formules exponentielles, in: Séminaire de Probabilités X (Seconde partie: Théorie des intégrales stochastiques, Strasbourg, 1974/1975), Lecture Notes in Math. 511, Springer, Berlin, 1976, 481-500.

Département de Mathématiques

Université de La Rochelle

Avenue Michel Crépeau

17042 La Rochelle Cedex 1, France

E-mail: yelkhati@univ-lr.fr

nprivaul@univ-lr.fr

Received on 27.6.2002;

revised version on 11.2.2003 